\title{
A massively parallel and memory \\ efficient FEM toolbox with A Hybrid Total FETI solver with accelerator support
}

\author{
Lubomir Riha1, Michal Merta ${ }^{1,2}$, Radim Vavrik ${ }^{1}$, Tomas Brzobohaty ${ }^{1}$, Alexandros \\ Markopoulos $^{1}$, Ondrej Meca ${ }^{1}$, Ondrej Vysocky ${ }^{1}$, Tomas Kozubek ${ }^{1}$ and Vit Vondrak ${ }^{1}$
}

\begin{abstract}
In this paper we present the ESPRESO FEM library, which includes a FEM toolbox with interfaces to professional and open-source simulation tools, and a massively parallel Hybrid Total FETI (HTFETI) solver which can fully utilize the OLCF Titan supercomputer, and achieves super-linear scaling. This paper presents several new techniques for FETI solvers designed for efficient utilization of supercomputers with a focus on: (i) performance - we present a fivefold reduction of solver runtime for the Laplace equation by redesigning the FETI solver, and offloading the key workload to the accelerator. We compare Intel Xeon Phi 7120p and Tesla K80 and P100 accelerators to Intel Xeon E5-2680v3 and Xeon Phi 7210 CPUs; and (ii) memory efficiency - we present two techniques which increase the efficiency of the HTFETI solver 1.8 times, and pushes the limits of the largest possible problem ESPRESO can solve from 124 to 223 billion unknowns for problems with unstructured meshes. Finally we show that by dynamicly tuning hardware parameters we can reduce energy consumption by up to $33 \%$.
\end{abstract}

\section{Keywords}

FEM, Hybrid Total FETI, GPU, Xeon Phi, KNC, KNL, massively parallel, sparse linear solver

\section{Introduction}

In this paper we present the ExaScale PaRallel FETI SOlver (ESPRESO) Finite Element Method (FEM) library which contains (i) a FEM preprocessor (ESPRESO FEM) and (ii) a Finite Element Tearing and Interconnecting (FETI) solver. The ESPRESO FEM preprocessor requires a mesh generated by 3rd party software together with definition of groups of elements, we call them sets, onto which boundary conditions are prescribed. The boundary conditions are described by an ESPRESO configuration file. We support the input formats of one commercial and two open-source tools for mesh and sets data. The commercial tool is ANSYS Workbench (ANSYS, 2017) and the open-source tools are ELMER (IT Center for Science, CSC, 2017), and OpenFOAM (Jasak et al., 2007).

When using the standard toolchain, the ESPRESO FEM assembles all FEM matrix objects and passes them to the FETI solver. As of now the ESPRESO FEM contains assemblers for structural mechanics (heat transfer and linear elasticity) problems, but new physics are being continuously implemented. At the end, mesh and simulation results are stored in the VTK format (Visualization Toolkit), see Shroeder et al. (1998). Moreover, in collaboration with ELMER developers at CSC - IT Center for Science, we have developed an API which transfers all necessary FEM objects. In this case ESPRESO serves only as a massively parallel linear solver.

The interface that allows our library to connect with other FEM tools enables our scalable solvers to be used by the listed tools. The solver also supports modern many-core architectures, the Intel Xeon Phi and the NVIDIA GPU, using methods described in Section 3. Section 4.2 then presents solver performance.

One of the key components of the library is the massively parallel linear solver. It is based on the Finite Element Tearing and Interconnecting method (FETI, for more detail see, e.g., in Farhat et al. (1994a); Gosselet and Rey (2006)) which are successfully used in the engineering community for very large problems.

In this method the original structure is decomposed into several non-overlapping subdomains. Mutual continuity of primal variables between neighboring subdomains is enforced afterwards by dual variables, i.e., Lagrange multipliers (LM). They are usually obtained iteratively through certain Krylov subspace methods, then the primal solution is evaluated locally on each subdomain. Dostál et al. (2006) introduced a new variant of an algorithm called Total FETI (TFETI) in which the Dirichlet boundary condition is enforced by LM as well.

ESPRESO contains both the original TFETI method as well as its variant called HTFETI (Hybrid TFETI). The HTFETI method is a variant of hybrid FETI methods for FETI and FETI-DP, introduced by Klawonn and Rheinbach (2010). In this approach a number of subdomains is

\footnotetext{
${ }^{1}$ IT4Innovations National Supercomputing Center, VSB-Technical University of Ostrava, CZ

${ }^{2}$ Dept. of Applied Mathematics, VSB-Technical University of Ostrava, CZ
}

\section{Corresponding author:}

Lubomir Riha, IT4Innovations National Supercomputing Center, VSBTechnical University of Ostrava, 17. listopadu 15/2172, Ostrava, Czech Republic

Email: lubomir.riha@vsb.cz 
combined into clusters, which can be seen as a three-level domain decomposition approach.

The main advantage of HTFETI is its potential to solve problems decomposed into a large number of subdomains due to reduction of the main bottleneck of the FETI methods, the coarse problem. In this paper we show that our implementation in one of the presented examples uses 81.2 million subdomains organized into 17,576 clusters processed by the 17,576 compute nodes of the Titan supercomputer installed in the Oak Ridge Leadership Computing Facility, OLCF (2017). This means that: (i) the solver is able to solve extremely large problems (over 223 billion unknowns for the 3D Laplace equation); (ii) the solver can decompose smaller problems of approximately 1 billion unknowns into small subdomains, which improves memory efficiency (faster Cholesky factorization of small matrices) and the performance of the solver in terms of the FETI numerical scalability (a smaller number of iterations).

To further improve the memory efficiency of our HTFETI solver we propose two techniques: (i) a repeated factorization technique and (ii) an iterative solver technique to solve subdomain stiffness matrices. Both techniques eliminate the high memory utilization caused by the LU or Cholesky decomposition done by the sparse direct solvers that are used in all FETI solvers. The problems presented in this paper use unstructured meshes, and a significant part of the memory is therefore used by the mesh and the stiffness matrices.

Scalable implementations of domain decomposition methods have been presented in several works. Klawonn et al. (2016b) combined FETI-DP methods with a parallel algebraic multigrid as a solver for the coarse problem, to increase the scalability. The authors focused on 2D linear elasticity and nonlinear hyperelasticity problems, and demonstrated scalability to more than 10 billion DOFs and 500,000 MPI ranks. The total memory consumption was approximately $256 \mathrm{~TB}$. In comparison, we present a 3D linear elasticity problem with 70 billion unknowns using 560 TB of memory. Similarly, Klawonn et al. (2016a) presented a solution used micro-macro scale bridging in the simulation of dual-phase steel. The 3D solver is able to scale up to 786,432 cores, solving problems with more than 19 billion unknowns, using 768 TB of memory.

Highly scalable implementations of the Balancing Domain Decomposition by Constraint (BDDC) method are presented in Badia et al. (2014) and Sistek and Cirak (2015). The former paper outlines the solution of 3D Poisson and linear elasticity problems with up to 1 billion unknowns on 27 thousand cores consuming 110 TB of RAM. The latter focuses on the simulation of low Reynolds number flows around fixed and moving rigid bodies in 3D. The largest solved problem, reaching 3.3 billion unknowns, was solved using 65,500 cores and 65 TB of memory. These results demonstrate the abilities of non-overlapping domain decomposition methods to utilize computational resources of current multi-petascale supercomputers for solution of various kinds of problems.

However, large numbers of supercomputers are now equipped with many-core accelerator technologies. Therefore, in addition to the scalable HTFETI implementation, the paper presents acceleration using the Intel Xeon Phi coprocessors or GPU accelerators, which enables us to fully utilize

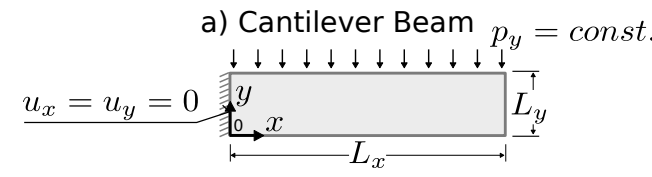

b) Size of the coarse problem - \#Rigid Body Motions 12 for TFETI 6 for HTFETI
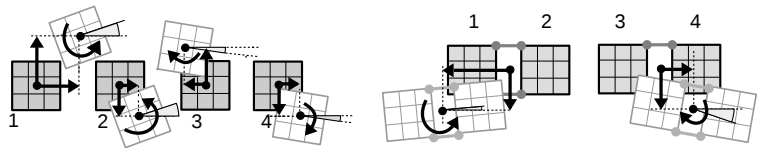

Figure 1. A cantilever beam in 2D.

the computational power of these accelerated machines; see the list in Table 2.

FETI methods are based on sparse matrices, and the most time consuming operation is the solve routine of the sparse direct solver. This is called for all subdomain matrices repeatedly in every iteration of the Preconditioned Conjugate Projected Gradient (PCPG) solver. We present two approaches to accelerating this operation: (i) maintain the sparse matrices by using a sparse direct solver on the accelerator; and (ii) use our proposed approach, which converts sparse data to dense. Then instead of using the solve routine on sparse data, the dense general matrix-vector (GEMV) multiplication operation is used. The size of the new dense matrix is similar to the size of the Cholesky decomposition of the original sparse matrix.

The first approach can be used efficiently for Intel Xeon Phi, both the Knights Corner (KNC) and the Knights Landing (KNL) generations, since the MKL PARDISO sparse direct solver is well optimized for these. However for GPUs we have not found any suitable alternative. The second approach converts the sparse data into dense using the local Schur complement (LSC) method for HTFETI, presented in the Section '2.2' which was originally presented for Total FETI in Riha et al. (2016b). The main advantage of the LSC method is that GEMV operation provides regular memory access and is therefore able to fully utilize the high memory bandwidth of the accelerator.

\section{Methods}

In this section we introduce the Hybrid Total FETI method, the key element for the scalability of the ESPRESO solvers, and the local Schur complement method as a key tool for efficient acceleration of the HTFETI method.

\subsection{Hybrid Total FETI method}

The principle of the HTFETI method is explained in contrast to the classical TFETI method.

2.1.1 Total FETI method We will shortly introduce the TFETI method for a two-dimensional linear elasticity problem given by a cantilever beam (see Figure 1.a). After discretization and domain decomposition, the $i$-th set of nodal equilibrium equations related to $i$-th subdomain is

$$
\mathbf{K}_{i} \mathbf{u}_{i}=\mathbf{f}_{i}-\mathbf{B}_{i}^{\top} \boldsymbol{\lambda} \quad i \in(1,2,3,4)
$$

where $\mathbf{K}_{i}$ is the local subdomain stiffness matrix and $\mathbf{f}_{i}$ is the local right-hand side vector, both are assembled only 
from elements belonging to $i$-th subdomain. $\boldsymbol{\lambda}$ is a vector of LMs (reaction forces enforcing the continuity between neighbouring subdomains) and $\mathbf{B}_{i}$ is the constraints matrix. These four systems of linear equations are complemented by the compatibility condition

$$
\mathbf{B}_{1} \mathbf{u}_{1}+\mathbf{B}_{2} \mathbf{u}_{2}+\mathbf{B}_{3} \mathbf{u}_{3}+\mathbf{B}_{4} \mathbf{u}_{4}=\mathbf{c}
$$

All of these local matrices $\mathbf{B}_{i}$ appearing in (2) can be collected in one global object

$$
\mathbf{B}=\mathbf{B}_{1: 4}=\left(\begin{array}{llll}
\mathbf{B}_{1} & \mathbf{B}_{2} & \mathbf{B}_{3} & \mathbf{B}_{4}
\end{array}\right)
$$

which enforces the continuity on the decomposed structure, and also the Dirichlet boundary condition. Introducing the block diagonal matrix $\mathbf{K}=\mathbf{K}_{1: 4}=$ $\operatorname{diag}\left(\mathbf{K}_{1}, \mathbf{K}_{2}, \mathbf{K}_{3}, \mathbf{K}_{4}\right)$, the global right-hand side vector $\mathbf{f}^{\top}=\mathbf{f}_{1: 4}^{\top}=\left(\mathbf{f}_{1}^{\top}, \mathbf{f}_{2}^{\top}, \mathbf{f}_{3}^{\top}, \mathbf{f}_{4}^{\top}\right)$, and the global vector of

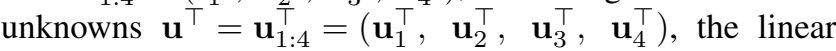
system representing the discretized equilibrium equations of the cantilever beam can be written as follows:

$$
\left(\begin{array}{ll}
\mathbf{K} & \mathbf{B}^{\top} \\
\mathbf{B} & \mathbf{O}
\end{array}\right)\left(\begin{array}{l}
\mathbf{u} \\
\lambda
\end{array}\right)=\left(\begin{array}{l}
\mathbf{f} \\
\mathbf{c}
\end{array}\right)
$$

In the text above, we also introduced the interval selection of matrices and vectors. For instance, $\mathbf{K}_{i: j}$ means the block diagonal stiffness matrix containing local stiffness matrices of subdomains number $i, i+1, i+2, \ldots, j$.

Eliminating primal variable $\mathbf{u}$ by

$$
\mathbf{u}=\mathbf{K}^{+}\left(\mathbf{f}-\mathbf{B}^{\top} \boldsymbol{\lambda}\right)+\mathbf{R} \boldsymbol{\alpha}
$$

and utilizing the orthogonality condition

$$
\mathbf{R} \perp \mathbf{f}-\mathbf{B}^{\top} \boldsymbol{\lambda}
$$

we arrive at the Schur complement system

$$
\left(\begin{array}{ll}
\mathbf{F} & \mathbf{G} \\
\mathbf{G}^{\top} & \mathbf{O}
\end{array}\right)\left(\begin{array}{l}
\boldsymbol{\lambda} \\
\boldsymbol{\alpha}
\end{array}\right)=\left(\begin{array}{l}
\mathbf{d} \\
\mathbf{e}
\end{array}\right),
$$

where $\mathbf{F}=\mathbf{B K}^{+} \mathbf{B}^{\top}$ is the TFETI operator, $\mathbf{G}=-\mathbf{B R}$ is a transformation matrix, and $\mathbf{d}=\mathbf{B} K^{+} \mathbf{f}-\mathbf{c}$ and $\mathbf{e}=$ $-\mathbf{R}^{\top} \mathbf{f}^{\top}$ are parts of the dual right hand side vector. The matrix $\mathbf{R}=\mathbf{R}_{1: 4}=\operatorname{diag}\left(\mathbf{R}_{1}, \mathbf{R}_{2}, \mathbf{R}_{3}, \mathbf{R}_{4}\right)$ consists of the vectors that create the basis of the null space of the stiffness matrix K. From the statics point of view, the basis stored in matrix $\mathbf{R}$ describes rigid body movements (RBM) of all subdomains. The vector $\boldsymbol{\alpha}$ contains their amplitudes.

It is worth noting that the new system (7) is better conditioned than (4), and it will be solved by the PCPG method. In the algorithm, the gradient is projected at each iteration by the orthogonal projector

$$
\mathbf{P}=\mathbf{I}-\mathbf{G}\left(\mathbf{G}^{\top} \mathbf{G}\right)^{-1} \mathbf{G}^{\top}
$$

where $\mathbf{G}=-\mathbf{B R}$. The part of the projector $\mathbf{G}^{\top} \mathbf{G} \in$ $\mathbb{R}^{n_{G} \times n_{G}}$ called the coarse problem is usually orthogonalized or factorized to improve the performance. Its dimension

$$
n_{G}=n_{s u b} \cdot d
$$

proportionally depends on the number of subdomains $n_{s u b}$. The parameter $d$ (number of RBMs of a single floating subdomain) is 3 in $2 \mathrm{D}$ and 6 in $3 \mathrm{D}$. If the number of subdomains is too large, the coarse problem can be a serious bottleneck for the TFETI method. Concretely for the simple beam benchmark (see Figure 1.b left), the size is given by

$$
n_{G}=(4 \text { subdomains }) \times(3 \mathrm{RBM})=12 .
$$

The possible ways to overcome it will be shown in the next section.

\subsubsection{The HTFETI method We apply the HTFETI method} to the same problem in order to demonstrate how the size of the coarse problem can be decreased. Firstly, we introduce the new object

$$
\mathbf{B}_{c}=\left(\begin{array}{ll}
\mathbf{B}_{c, 1: 2} & \mathbf{O} \\
\mathbf{O} & \mathbf{B}_{c, 3: 4}
\end{array}\right)=\left(\begin{array}{llll}
\mathbf{B}_{c, 1} & \mathbf{B}_{c, 2} & \mathbf{O} & \mathbf{O} \\
\mathbf{O} & \mathbf{O} & \mathbf{B}_{c, 3} & \mathbf{B}_{c, 4}
\end{array}\right)
$$

similar to the constraint matrix $\mathbf{B}$. The first diagonal block $\mathbf{B}_{c, 1: 2}$ consists of constraints that lock all mutual rigid movements between subdomains number one and two. This group will be denoted as cluster number one. The second block $\mathbf{B}_{c, 3: 4}$, locks mutual movements between subdomains number three and four (see Figure 1.b right). The block structure of $\mathbf{B}_{c}$ will be utilized in an algorithm later on. The cluster of subdomains in the HTFETI method can be viewed in the same manner as a single subdomain in the classical TFETI method. The collection of LMs that enforce the required locking effect is denoted as $\tilde{\boldsymbol{\lambda}}_{c}^{\top}=\left(\tilde{\boldsymbol{\lambda}}_{c, 1}^{\top}, \tilde{\boldsymbol{\lambda}}_{c, 2}^{\top}\right)$.

The extended linear system (nodal equilibrium equations of the cantilever beam with additional constraints) can be written as follows:

$$
\left(\begin{array}{lll}
\mathbf{K} & \mathbf{B}_{c}^{\top} & \mathbf{B}^{\top} \\
\mathbf{B}_{c} & \mathbf{O} & \mathbf{O} \\
\mathbf{B} & \mathbf{O} & \mathbf{O}
\end{array}\right)\left(\begin{array}{c}
\mathbf{u} \\
\boldsymbol{\lambda}_{c} \\
\boldsymbol{\lambda}
\end{array}\right)=\left(\begin{array}{l}
\mathbf{f} \\
\mathbf{o} \\
\mathbf{c}
\end{array}\right)
$$

and the choice of $\mathbf{B}_{c}$ is realized in a way that the primal solution $\mathbf{u}$ is the same as $\mathbf{u}$ in the original (4). In other words, the additional set of constraints in $\mathbf{B}_{c}$ does not violate the primal solution linked to constraints stored in $\mathbf{B}$. Thanks to the block-structure of $\mathbf{B}_{c}$, the system may be reordered according to clusters 1 and 2 into

$$
\left(\begin{array}{lll|lll|l}
\mathbf{K}_{1} & \mathbf{O} & \mathbf{B}_{c, 1}^{\top} & \mathbf{O} & \mathbf{O} & \mathbf{O} & \mathbf{B}_{1}^{\top} \\
\mathbf{O} & \mathbf{K}_{2} & \mathbf{B}_{c, 2}^{\top} & \mathbf{O} & \mathbf{O} & \mathbf{O} & \mathbf{B}_{2}^{\top} \\
\mathbf{B}_{c, 1} & \mathbf{B}_{c, 2} & \mathbf{O} & \mathbf{O} & \mathbf{O} & \mathbf{O} & \mathbf{O} \\
\hline \mathbf{O} & \mathbf{O} & \mathbf{O} & \mathbf{K}_{3} & \mathbf{O} & \mathbf{B}_{c, 3}^{\top} & \mathbf{B}_{3}^{\top} \\
\mathbf{O} & \mathbf{O} & \mathbf{O} & \mathbf{O} & \mathbf{K}_{4} & \mathbf{B}_{c, 4}^{\top} & \mathbf{B}_{4}^{\top} \\
\mathbf{O} & \mathbf{O} & \mathbf{O} & \mathbf{B}_{c, 3} & \mathbf{B}_{c, 4} & \mathbf{O} & \mathbf{O} \\
\hline \mathbf{B}_{1} & \mathbf{B}_{2} & \mathbf{O} & \mathbf{B}_{3} & \mathbf{B}_{4} & \mathbf{O} & \mathbf{O}
\end{array}\right)\left(\begin{array}{c}
\mathbf{u}_{1} \\
\mathbf{u}_{2} \\
\boldsymbol{\lambda}_{c, 1} \\
\hline \mathbf{u}_{3} \\
\mathbf{u}_{4} \\
\boldsymbol{\lambda}_{c, 2} \\
\hline \boldsymbol{\lambda}
\end{array}\right)=\left(\begin{array}{c}
\mathbf{f}_{1} \\
\mathbf{f}_{2} \\
\mathbf{o} \\
\hline \mathbf{f}_{3} \\
\mathbf{f}_{4} \\
\mathbf{o} \\
\hline \mathbf{c}
\end{array}\right)
$$

and consistently with the line partitioning rewritten into

$$
\left(\begin{array}{c|c|c}
\tilde{\mathbf{K}}_{1} & \mathbf{O} & \tilde{\mathbf{B}}_{1}^{\top} \\
\hline \mathbf{O} & \tilde{\mathbf{K}}_{2} & \tilde{\mathbf{B}}_{2}^{\top} \\
\hline \tilde{\mathbf{B}}_{1} & \tilde{\mathbf{B}}_{2} & \mathbf{O}
\end{array}\right)\left(\begin{array}{c}
\tilde{\mathbf{u}}_{1} \\
\hline \tilde{\mathbf{u}}_{2} \\
\hline \tilde{\boldsymbol{\lambda}}
\end{array}\right)=\left(\begin{array}{c}
\tilde{\mathbf{f}}_{1} \\
\tilde{\tilde{\mathbf{f}}}_{2} \\
\hline \tilde{\mathbf{c}}
\end{array}\right) .
$$

Clearly, $\boldsymbol{\lambda}=\tilde{\boldsymbol{\lambda}}$ and $\mathbf{c}=\tilde{\mathbf{c}}$. One can interpret the system (14) as a problem solved by the TFETI method with two subdomains where matrices $\tilde{\mathbf{K}}_{1}$ and $\tilde{\mathbf{K}}_{2}$ are the 
corresponding (cluster) stiffness matrices. Denoting $\tilde{\mathbf{K}}=$ $\operatorname{diag}\left(\tilde{\mathbf{K}}_{1}, \tilde{\mathbf{K}}_{2}\right), \tilde{\mathbf{B}}=\left(\tilde{\mathbf{B}}_{1}, \tilde{\mathbf{B}}_{2}\right), \tilde{\mathbf{f}}^{\top}=\left(\tilde{\mathbf{f}}_{1}^{\top}, \tilde{\mathbf{f}}_{2}^{\top}\right)$, and $\tilde{\mathbf{u}}^{\top}=$ $\left(\tilde{\mathbf{u}}_{1}^{\top}, \tilde{\mathbf{u}}_{2}^{\top}\right)$, the system (14) is simplified into

$$
\left(\begin{array}{cc}
\tilde{\mathbf{K}} & \tilde{\mathbf{B}}^{\top} \\
\tilde{\mathbf{B}} & \mathbf{O}
\end{array}\right)\left(\begin{array}{c}
\tilde{\mathbf{u}} \\
\tilde{\lambda}
\end{array}\right)=\left(\begin{array}{c}
\tilde{\mathbf{f}} \\
\tilde{\mathbf{c}}
\end{array}\right) .
$$

Using the steps and substitutions (here with tilde) applied in (4), (5), (6), and (7), we derive the dual Schur complement also for the HTFETI method in the form

$$
\left(\begin{array}{ll}
\tilde{\mathbf{F}} & \tilde{\mathbf{G}} \\
\tilde{\mathbf{G}}^{\top} & \mathbf{O}
\end{array}\right)\left(\begin{array}{c}
\tilde{\lambda} \\
\tilde{\boldsymbol{\alpha}}
\end{array}\right)=\left(\begin{array}{c}
\tilde{\mathbf{d}} \\
\tilde{\mathbf{e}}
\end{array}\right)
$$

which is solvable by the same iterative solvers as in the TFETI method case. However the current coarse problem

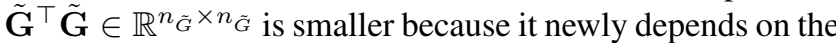
number of clusters and not of subdomains. With the same meaning of parameter $d$ as in (10), the size is given by

$$
n_{\tilde{G}}=(2 \text { clusters }) \times(3 \mathrm{RBM})=6,
$$

as illustrated in Figure 1.b right. Obviously, the beam problem used as an example does not contain a large number of subdomains. Nevertheless, it is 'large' enough to demonstrate how the size of the coarse problem is managed by the HTFETI method.

However, the HTFETI method does not bring only positive changes. For the same discretized and decomposed problem, the assembled system of linear equations in the HTFETI method is not as well-posed as the one assembled for the TFETI method. Therefore, one can expect the number of iterations to be slightly higher.

There are at least two important points that should be mentioned in contrast to the original TFETI method:

First, the orthogonal projection by $\tilde{\mathbf{P}}=\mathbf{I}-$ $\tilde{\mathbf{G}}\left(\tilde{\mathbf{G}}^{\top} \tilde{\mathbf{G}}\right)^{-1} \tilde{\mathbf{G}}^{\top}$ in the HTFETI case can be performed for the same number of subdomains efficiently, due to the locking effect of specific RBM (the coarse problem requires less memory for its storage and maintenance).

Second, the application of the generalized inverse of the stiffness matrix $\tilde{\mathbf{K}}$ (for more details see, e.g., Riha et al. (2016a)), the most expensive operation appearing in the operator $\tilde{\mathbf{F}}$, contains additional operations. To see the differences, the application of the HTFETI operator (matrix vector product)

$$
\mathbf{v}=\tilde{\mathbf{F}} \mathbf{p}=\sum_{I=1}^{n_{\text {clust }}} \tilde{\mathbf{F}}_{I} \mathbf{p}=\sum_{I=1}^{n_{\text {clust }}} \tilde{\mathbf{B}}_{I} \tilde{\mathbf{K}}_{I}^{+} \tilde{\mathbf{B}}_{I}^{\top} \mathbf{p}
$$

written with the objects introduced for the TFETI method plus $\mathbf{B}_{c}$ is

$$
\mathbf{v}=\sum_{I=1}^{n_{\text {clust }}} \underbrace{\underbrace{\mathbf{B}_{j: k} \mathbf{K}_{j: k}^{+} \mathbf{B}_{j: k}^{\top} \mathbf{p}}_{\text {TFETI }}+\mathbf{B}_{j: k} \mathbf{R}_{j: k} \boldsymbol{\beta}_{I}-\mathbf{B}_{j: k} \mathbf{K}_{j: k}^{+} \mathbf{B}_{c, j: k}^{\top} \boldsymbol{\mu}_{I}}_{\text {HTFETI }} .
$$

The first product is the same as in the case of the classsical TFETI method. The only difference is in the second and third products, which relate to the clusters. The meaning of $\boldsymbol{\beta}_{I}$ and $\boldsymbol{\mu}_{I}$ is described together with the special Schur complement $\mathbf{S}_{c, I}=\mathbf{G}_{c, I}^{\top} \mathbf{F}_{c, I}^{-1} \mathbf{G}_{c, I}$ in more detail, e.g., in Riha et al. (2016a). It is worth noting that always only one triplet of $\boldsymbol{\beta}_{I}, \boldsymbol{\mu}_{I}$ and $\mathbf{S}_{c, I}$ relates to one cluster.

\subsection{The local Schur complement method}

TFETI solver processing can be divided into two stages: (i) preprocessing and (ii) solver run time. During the preprocessing stage the most time consuming tasks include assembling the distributed inverse matrix of the coarse problem $\left(\mathbf{G}^{\top} \mathbf{G}\right)^{-1}=\left(\mathbf{R}^{\top} \mathbf{B}^{\top} \mathbf{B R}\right)^{-1}$ and factorization of the subdomain stiffness matrices $\hat{\mathbf{K}}_{i}$. While the coarse problem processing time is mainly given by the number of subdomains, the $\hat{\mathbf{K}}_{i}$ factorization times are given by the subdomain sizes. The solver run time stage executes the PCPG method and its execution time is therefore given by the number of iterations and the iteration time. The most time consuming part of a single iteration is the execution of the solve routine (forward and backward substitutions) of the sparse direct solver using the Cholesky decomposition of the $\hat{\mathbf{K}}_{i}$. This operation can take up to $95 \%$ of the iteration time (more detail can be found in Riha et al. (2016a)). The main focus of this paper is to describe a method to speed up this operation using accelerators.

Sparse data structures cannot take full advantage of accelerators which are equipped with high number of single instruction multiple data (SIMD) units. To reach the full potential of such architectures, a dense representation of sparse stiffness matrices $\hat{\mathbf{K}}_{i}$ and their Cholesky decomposition is required.

In the TFETI solver, prior to calling the solve routine with $\hat{\mathbf{K}}_{i}$, the vector of the LMs (the dual variables $\boldsymbol{\lambda}$ ) must be converted to primal variables using the gluing matrix $\mathbf{B}^{\top}$. The result of the solve routine (primal variables) has to again be converted into dual variables using $\mathbf{B}$. This operation appears during the initialization stage of the PCPG and then once at each iteration (in the regular conjugate gradient method it is equivalent to the matrix-vector multiplication with the system matrix). It contains two calls of sparse matrix vector multiplication from sparse BLAS (SpMV with $\mathbf{B}_{i}$ ), and one call of the solve routine of a sparse direct solver (e.g., the PARDISO solver (Kuzmin et al., 2013; Schenk et al., $2008,2007)$ ) - the action of generalized inverse $\mathbf{K}^{+}$. Instead of executing these three operations on sparse matrices, we can directly assemble the $i$-th contribution $\mathbf{F}_{i}$ to the global TFETI operator $\mathbf{F}$ from

$$
\mathbf{A}_{i}=\left(\begin{array}{cc}
\hat{\mathbf{K}}_{i} & \mathbf{B}_{i}^{\top} \\
\mathbf{B}_{i} & \mathbf{0}
\end{array}\right)
$$

by eliminating the primal variables and assembling the negative Schur complement $\mathbf{F}_{i}=\mathbf{B}_{i} \mathbf{K}_{i}^{+} \mathbf{B}_{i}^{\top}=\mathbf{B}_{i} \hat{\mathbf{K}}_{i}^{-1} \mathbf{B}_{i}^{\top *}$. In (20), the matrix $\hat{\mathbf{K}}_{i}$ is appropriately modified to $\mathbf{K}_{i}$, satisfying the equality $\hat{\mathbf{K}}_{i}^{-1}=\mathbf{K}_{i}^{+}$. We call this approach for replacing sparse operations with a multiplication by a single dense matrix the local Schur complement method.

An efficient approach to assemble the $\mathbf{F}_{i}$ matrix is to use an incomplete factorization method implemented in the PARDISO solver. When the factorization is stopped after the elimination of the $(1,1)$ block of the system in (20), the Schur complement $-\mathbf{B}_{i} \hat{\mathbf{K}}_{i}^{-1} \mathbf{B}_{i}^{\top}=-\mathbf{F}_{i}$ will be available in the block $(2,2)$. The numerical experiments presented in Table 4 show that the assembly of the $\mathbf{F}_{i}$

${ }^{*}$ Please note that when assembling the Schur complement the matrix $\mathbf{B}_{i}$ is used in its "condensed" form (only the non-zero rows are kept) 
matrix is significantly slower than the factorization of the stiffness matrix. On the other hand, the multiplication by the dense Schur complement matrix on accelerators with high bandwidth memory is significantly faster than the sparse solve on the CPU. Before using the method, it is therefore necessary to evaluate the tradeoff between the preprocessing time which is executed just once, and the iterative solver run time in which the local Schur complements are processed repeatedly in every iteration. These results are presented in Tables 3 and 4 .

2.2.1 The local Schur complement method for the HTFETI Operator In the previous section a method to accelerate the TFETI operator is described. However, in this paper we use primarily the HTFETI method. The application of the HTFETI operator is more complex, as shown in (19), and in order to get the contribution $\tilde{\mathbf{F}}_{I} \mathbf{p}$, the auxiliary variables $\boldsymbol{\beta}_{I}$ (rigid body motions amplitudes) and $\boldsymbol{\mu}_{I}(\mathrm{LM})$ have to be calculated at each iteration. It requires two additional factorizations of objects; $\mathbf{F}_{c, I}$ and $\mathbf{S}_{c, I}$ per each cluster in the preprocessing.

The non-negligible feature of the HTFETI method is that the first product $\mathbf{B}_{j: k} \mathbf{K}_{j: k}^{+} \mathbf{B}_{j: k}^{\top} \mathbf{p}$ in (19) can be executed on the accelerator (as described in the next section) independently of the remaining parts in (19), which can be performed concurrently on the CPU. The set of Schur complements $(j, j+1, \cdots, k)$ belonging to the $I$-th cluster denoted as

$$
\mathbf{F}_{I}=\sum_{i=j}^{k} \mathbf{F}_{i}=\mathbf{B}_{j: k} \mathbf{K}_{j: k}^{+} \mathbf{B}_{j: k}^{\top}=\mathbf{B}_{j: k} \hat{\mathbf{K}}_{j: k}^{-1} \mathbf{B}_{j: k}^{\top}
$$

is obtained one by one, the same way as in the TFETI method using (20) .

\section{Acceleration of the HTFETI Solver}

In this section we present two approaches for improving HTFETI solver performance using accelerators. The first approach employs a sparse direct solver running on the accelerator, the second, and more suitable one, uses the LSC method.

\subsection{Acceleration approaches}

As described in Section 2, we need to solve linear systems with symmetric positive definite matrices. There are only a few suitable sparse direct solvers which support accelerators as required by HTFETI method. For the Intel Xeon Phi (Jeffers and Reinders, 2013, 2014, 2015), we use the Intel version of the PARDISO solver (Kuzmin et al., 2013; Schenk et al., 2008, 2007), which is included in the Intel Math Kernel Library (MKL (Intel Corporation, 2003-2017)). The solver is optimized for many-core coprocessor architecture by the vendor. For the GPU accelerators, there are, e.g., the SuperLU library, which is suitable for nonsymetric systems only, and the SuiteSparse (Rennich et al., 2016) and PaStiX (Casadei et al., 2008-2017) libraries. These unfortunately provide acceleration for the factorization stage only. To utilize the GPU accelerators in HTFETI we need acceleration of the solve stage as well. Therefore the only option we found is the NVIDIA cuSolver library, which however is not yet optimized from both the performance and memory utilization points of view. The library is part of the CUDA toolkit, documented by NVIDIA Corporation (2006-2017).

\subsubsection{Direct sparse solve on Intel Xeon Phi (KNC) using} MKL PARDISO To accelerate the TFETI and HTFETI computation one can offload the sparse solve with the subdomain stiffness matrices $\hat{\mathbf{K}}_{i}$ during the application of the operators $\mathbf{B}_{i} \hat{\mathbf{K}}_{i}^{-1} \mathbf{B}_{i}^{\top}$ to the coprocessor. This consists of several steps:

1. During the preprocessing, after the subdomain stiffness matrices are assembled, their CSR data are collected into three contiguous arrays to minimize the number of individual data transfers via the PCIExpress bus.

2. The data are offloaded to a coprocessor. Additional memory is allocated on the device (for input/output vectors, sparse solver auxiliary arrays, etc.)

3. Matrices are factorized on the coprocessor.

4. Within each iteration, the right hand side (RHS) vectors are collected into a single array and offloaded to the device, where the solve is performed within an OpenMP parallel region with up to 244 threads.

\subsubsection{Direct sparse solve on NVIDIA GPU using} cuSolver In the preprocessing stage of the ESPRESO solver, a new instance of the sparse cuSolver context is initialized for each subdomain. To overlap the kernel execution and data transfers, a set of CUDA streams is created, and each stream is associated with group of subdomains. After the setup of the cuSolver context, the reordering of the $\hat{\mathbf{K}}_{i}$ matrix using the Symmetric Approximate Minimum Degree Algorithm (SYMAMD (Amestoy et al., 1996)) provided by the cuSolver library is performed. This operation reduces the zero fill-in during the following symbolic and numeric Cholesky factorization. While reordering of the matrix is performed by the CPU, the factors are computed using the GPU accelerator and kept in the GPU memory.

In each PCPG iteration the RHS vector is reordered by the CPU and transferred to the GPU memory. The solve routine of the cuSolver is executed on the GPU. The solution vector is transferred back to the host memory and reordered. Due to the CUDA streams, everything is asynchronous with CPU processing.

3.1.3 The local Schur complement method The main disadvantage of the approach presented in the previous section is the usage of sparse data structures, which leads to an irregular memory access pattern. To make use of the full potential of the many-core architecture we use the local Schur complement method presented in Section 2.2. This enables us to replace the sparse solve with dense matrixvector multiplication within each iteration of the PCPG solver. The workflow is similar to the case of the sparse solve (see Figure 2). The following steps differ according to the architecture of the accelerator.

Knights Corner coprocessors In the case of the Knights Corner coprocessor, the matrices of Schur complement $\mathbf{F}_{i}=$ $\mathbf{B}_{i} \mathbf{K}_{i}^{+} \mathbf{B}_{i}^{\top}=\mathbf{B}_{i} \hat{\mathbf{K}}_{i}^{-1} \mathbf{B}_{i}^{\top}$ are assembled on the host using the PARDISO solver, stored in a contiguous array, and the array is then offloaded to the accelerator. 


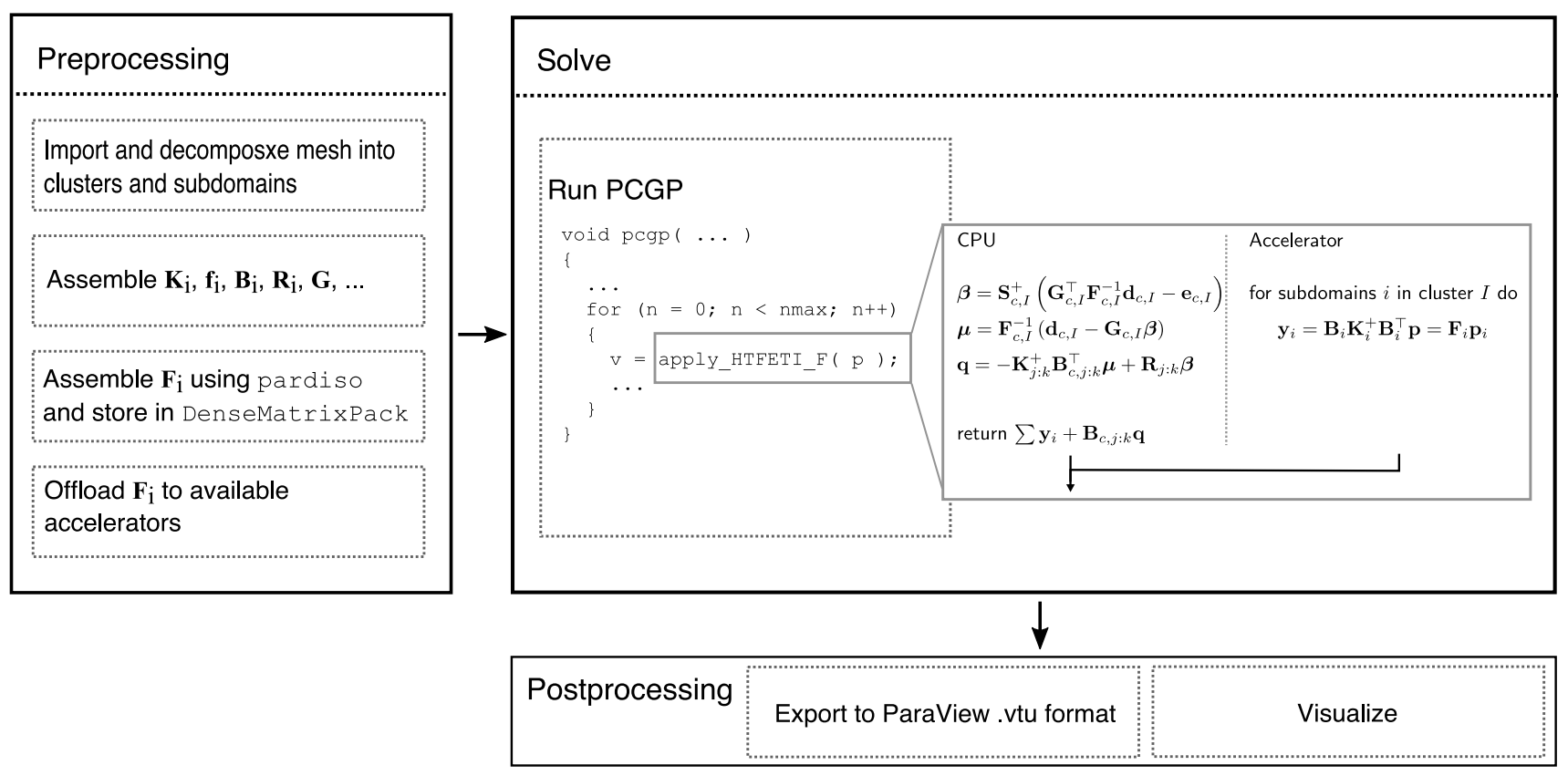

Figure 2. Acceleration using the local Schur complement method.

Within each iteration of the CG-based solver the multiplied vectors are collected into a single array and offloaded to the device. The dense matrix-vector multiplication is performed within an OpenMP parallel region on a device with up to 244 threads. The offloaded computation is done asynchronously, and at the same time the host performs its computation (i.e., application of additional HTFETI operators or processing of subdomains that do not fit into coprocessor's memory). After multiplication the results are sent back to the host within a single contiguous array.

GPU acceleration In the case of the GPU accelerator, the total size of Schur complement matrices is first computed. Considering the available amount of device memory, an appropriate number of subdomains are marked for processing on the GPU using the LSC method, while the rest of them remain on the CPU. According to the solver configuration, the subdomains on the CPU can be processed either using the LSC method, or with a sparse direct solver. The Schur complement $\mathbf{F}_{i}$ is then assembled for particular marked subdomains in parallel on the CPU, using the PARDISO solver.

To perform the fast symmetric matrix-vector multiplication using the cuBLAS library (NVIDIA Corporation, 20062017), and to use the device memory efficiently, we combine the Schur complements of the two neighboring subdomains in the following manner. If the size of $\mathbf{F}_{i}$ is greater than the size of $\mathbf{F}_{i+1}$, the upper triangular part of $\mathbf{F}_{i}$ is preserved in the original array, and the lower part is overwritten by the lower part of $\mathbf{F}_{i+1}$. In the opposite case, when the size of $\mathbf{F}_{i}$ is lower than the size of $\mathbf{F}_{i+1}$, the larger array with the lower triangular part of $\mathbf{F}_{i+1}$ is preserved and the upper part is overwritten by the upper part of $\mathbf{F}_{i}$. In cases where the size of both matrices is equal, a new larger array is allocated to fit both triangular matrices including their diagonals, and filled with the upper part of $\mathbf{F}_{i}$ and the lower part of $\mathbf{F}_{i+1}$.
The pointer to the first element of each triangular matrix and its leading dimension is stored along with the array and used during the cuBLAS method call. After the Schur complements are combined, the shared arrays are transferred to the GPU memory. The GPU memory and the pinned host memory is also preallocated for the corresponding vectors of the multiplication.

In each PCPG iteration, before the $\mathbf{F}_{i} \mathbf{p}_{i}$ multiplication, the $\mathbf{p}_{i}$ vector is transferred from the pinned host memory to the device memory using an asynchronous memcopy operation in a separate CUDA stream from a streams pool. The resulting vector is transferred back to the pinned host memory in an analogous manner.

In both cases, converting sparse data structures to dense ones enables us to use optimized and vectorized dense BLAS routines with a regular and coalesced memory access pattern. This is essential in order to be able to utilize accelerators' fast memories and wide SIMD units. As will be seen from the numerical experiments, the iteration time can be significantly reduced in comparison with the original CPU code. On the other hand, the preprocessing time is increased, since the Schur complement computation is more demanding than the factorization of the sparse matrix. This makes the method more suitable for problems requiring a large number of PCPG iterations, e.g. transient or ill-conditioned problems.

3.1.4 Acceleration of the preconditioner application The Dirichlet preconditioner (Farhat et al., 1994b) has the form

$$
\mathbf{F}^{D-1}=\sum_{s=1}^{N_{s}} \mathbf{B}_{s}\left(\begin{array}{cc}
\mathbf{0} & \mathbf{0} \\
\mathbf{0} & \mathbf{S}_{s}^{b b}
\end{array}\right) \mathbf{B}_{s}^{\top},
$$

where $\mathbf{S}_{s}^{b b}$ is a dense Schur complement matrix

$$
\mathbf{S}_{s}^{b b}=\mathbf{K}_{s}^{b b}-\left(\mathbf{K}_{s}^{i b}\right)^{\top}\left(\mathbf{K}_{s}^{i i}\right)^{-1} \mathbf{K}_{s}^{i b}
$$

Here $i$ are the indices of the local stiffness matrix $\mathbf{K}_{s}$ associated with interior degrees of freedom and $b$ represents 
Table 1. Acceleration of the Dirichlet preconditioner on Intel Xeon Phi 7120p. For comparison we also present the performance of the LSC method.

\begin{tabular}{lccc}
\hline & $\begin{array}{c}\text { Intel Xeon } \\
\text { E5-2680v3 time [s] }\end{array}$ & $\begin{array}{c}\text { Intel Xeon } \\
\text { Phi 7120p time [s] }\end{array}$ & speedup \\
\hline FETI operator & $0.125 \mathrm{~s}$ & $0.062 \mathrm{~s}$ & 2.03 \\
\hline Dirichlet prec. & $0.075 \mathrm{~s}$ & $0.043 \mathrm{~s}$ & 1.75 \\
\hline
\end{tabular}

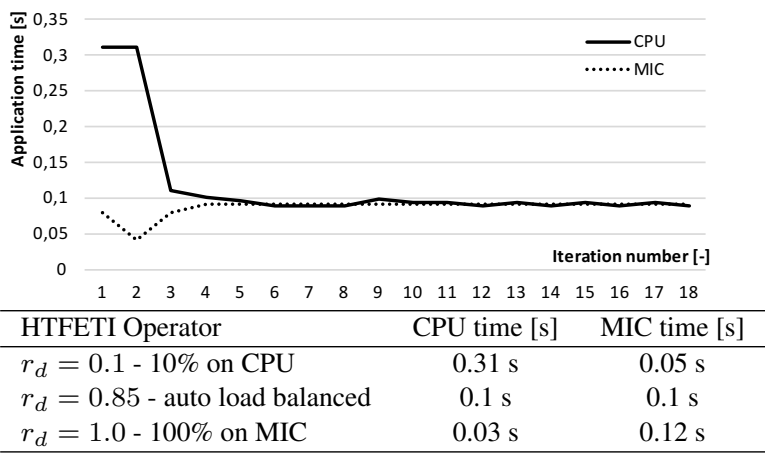

Figure 3. Load balancing of the iterative solver between the CPU (2x Xeon E2-2680v3) and the MIC (2x Xeon Phi 7120P)

indices of the boundary degrees of freedom. Since the matrix of the preconditioner is dense, it is suitable to offload its application to the coprocessor together with the action of the HTFETI operator. The preconditioner blocks are assembled using the host CPU during the preprocessing phase, stored in a contiguous array, and copied to the coprocessor. Then, during each iteration the gemv routine from the MKL is called to apply the preconditioner.

3.1.5 Load balancing While the offloaded computation is being performed, the host processor can simultaneously continue its work. It can perform tasks which are independent of the output of the coprocessor's computation (mainly in the case of the HTFETI method) or apply its portion of the blocks of the system matrix. The portion of matrix-vector multiplication processed by the coprocessor and host can easily be adjusted after every iteration using the following formula:

$$
r_{d}^{\text {new }}=\frac{r_{d}^{\text {old }} t_{h}}{r_{d}^{\text {old }} t_{h}+t_{d}\left(1-r_{d}^{\text {old }}\right)},
$$

where $r_{d}$ is the ratio of work done by the device and $t_{h}, t_{d}$ are computational times on the host and device respectively, in the previous iteration. In order to avoid expensive transfers of matrices between host and device after each iteration, the matrix data are duplicated on both. Using this simple approach we can automatically adjust workload distribution at the price of higher memory requirements.

\subsection{Architecture evaluation}

In Table 3 and Table 4 we present the processing times of the sparse direct solver and the local Schur complement method, comparing the selected architectures presented in Table 2. The problem sizes of the particular tests were selected to occupy the same amount of accelerator memory that was limited by the memory size of one NVIDIA Tesla K80 chip (12 GB). The key operations that are required by the HTFETI solver are presented. The following observations can be made from these tables: (i) MKL PARDISO on all Intel architectures performs significantly better than cuSolver on NVIDIA architectures; (ii) in the case of linear elasticity, the Xeon Phi 7210 is the fastest for both the factorization and solve, with a speedup of up to $2 \mathrm{x}$ with respect to the Xeon E5-2680v3, whereas in other cases, the sparse solvers on accelerators brought no speedup; (iii) the LSC method is fastest on the Tesla P100 with speedup up to $5.3 \mathrm{x}$ due to its high memory bandwidth. The difference of solve runtime follows the difference in memory bandwidth between the Tesla P100 and the Xeon Phi 7210, since the most time-consuming part performs the memory bound GEMV operation.

\section{Memory efficient implementation}

The most memory consuming objects of the FETI solvers in general are the Cholesky factors of the stiffness matrices $\mathbf{K}^{+}$. To eliminate these objects we propose two techniques in this section: (i) the repeated factorization technique and (ii) the iterative solver technique. Both techniques are able to reduce memory consumption for the cost of additional per iteration time of the PCPG solver.

\subsection{The repeated factorization technique}

This technique allows the user to select a set of subdomains for which the factorization is not performed during the preprocessing, but is performed within the action of the HTFETI operator. This means that in every PCPG iteration, both the factorization and the solve routine of MKL PARDISO are performed, and at the end the Cholesky decomposition objects are deleted. From a performance point of view the factorization is approximately 10 times slower for linear elasticity and 20 times slower for heat transfer than the solve routine. The effect of the repeated factorization is shown in Figure 4 where the number of kept factors goes from $100 \%$ (all factors are kept) to $85 \%$. The single iteration time increases from $1.66 \mathrm{~s}$ to $4.87 \mathrm{~s}$, and the amount of saved memory is $2.8 \mathrm{~GB}$. If one keeps no factors in the memory, the solver will save $16 \mathrm{~GB}$ of RAM and the single iteration time will be $15.7 \mathrm{~s}$ (factorization and solve runtime).

\subsection{The iterative solver technique}

The second technique uses the iterative CG solver instead of the sparse direct solver, therefore no preprocessing is required. The disadvantage of this method is that its performance is data dependent. The convergence of the iterative solver depends on the condition number of subdomain matrices. No preconditioner is used in order to keep the memory profile as low as possible.

Since the subdomain matrices for the 3D heat transfer (Laplace equation) are well conditioned, this method is more efficient for such problems than repeated factorization. Using this method we were able to solve the largest possible problem yet with our FEM package: 223 billion unknowns on the 17,573 compute nodes of Titan, approximately 12.8 million unknowns per compute node with 32 GB RAM. Please note that this is a problem with an unstructured mesh where assembled matrices are kept in the memory. In total 
Table 2. Key properties of the hardware architectures selected for the paper. Note: SP - single precision, DP - double precision; [1] only one GPU chip of the dual chip Tesla K80 accelerator; [2] PCI Express version of the Tesla P100 accelerator for PCle-based servers.

\begin{tabular}{|c|c|c|c|c|c|c|c|c|}
\hline & $\begin{array}{c}\text { Fabrication } \\
\text { process } \\
{[\mathrm{nm}]}\end{array}$ & $\begin{array}{c}\text { Clock } \\
\text { frequency } \\
{[\mathrm{GHz}]}\end{array}$ & $\begin{array}{l}\text { Number } \\
\text { of cores }\end{array}$ & $\begin{array}{c}\text { Peak floating } \\
\text { point performance } \\
\text { SP/DP [GFLOPS] }\end{array}$ & $\begin{array}{c}\text { Peak power } \\
\text { consumption } \\
\text { [W] }\end{array}$ & $\begin{array}{c}\text { Perf. per } \\
\text { Watt SP/DP } \\
\text { [GFLOPS/W] }\end{array}$ & $\begin{array}{c}\text { Theoretical } \\
\text { Memory } \\
\text { Bandwidth } \\
{[\mathrm{GB} / \mathrm{s}]}\end{array}$ & $\begin{array}{l}\text { Memory } \\
\text { type }\end{array}$ \\
\hline $\begin{array}{l}\text { Intel Xeon E5-2680v3 } \\
\text { (Intel Corporation, 2017a) }\end{array}$ & 22 & 2.5 & 12 & $960 / 480$ & 120 & $8.0 / 4.0$ & 68 & DDR4 \\
\hline $\begin{array}{l}\text { Intel Xeon Phi 7120p } \\
\text { (Intel Corporation, 2017b) }\end{array}$ & 22 & 1.24 & 61 & $2420 / 1210$ & 300 & $8.1 / 4.0$ & 352 & GDDR5 \\
\hline $\begin{array}{l}\text { Intel Xeon Phi 7210 } \\
\text { (Intel Corporation, 2017c) }\end{array}$ & 14 & 1.3 & 64 & 5325 / 2662 & 215 & $24.8 / 12.4$ & 102 / 400 & DDR4/MCDRAM \\
\hline $\begin{array}{l}\left.\text { NVIDIA Tesla K80 (1 chip }{ }^{[1]}\right) \\
\text { (NVIDIA Corporation, 2017b) }\end{array}$ & b) 22 & 0.56 & 1597 SP / 533 DP & $4365 / 1455$ & 150 & $29.1 / 9.7$ & 240 & GDDR5 \\
\hline $\begin{array}{l}\text { NVIDIA Tesla K20X (Titan) } \\
\text { (NVIDIA Corporation, 2017a) }\end{array}$ & a) 28 & 0.732 & 2688 SP / 896 DP & $3950 / 1310$ & 235 & $16.8 / 5.6$ & 250 & GDDR5 \\
\hline $\begin{array}{l}\text { NVIDIA Tesla P100 (PCle }{ }^{[2]} \text { ) } \\
\text { (NVIDIA Corporation, 2017c) }\end{array}$ & c) 16 & 1.328 & $3584 \mathrm{SP} / 1792 \mathrm{DP}$ & 8345 / 4217 & 250 & $33.4 / 16.9$ & 720 & HBM2 \\
\hline
\end{tabular}

Table 3. Performance of the sparse solve on various many-core architectures. Solve times are measured for 100 iterations of the PCPG solver. Note: [1] only one GPU chip of the dual chip Tesla K80 accelerator is used; [2] values for the Tesla P100 are extrapolated due to the excessive memory consumption of the cuSolver; [3] with respect to MKL PARDISO sparse solve on CPU.

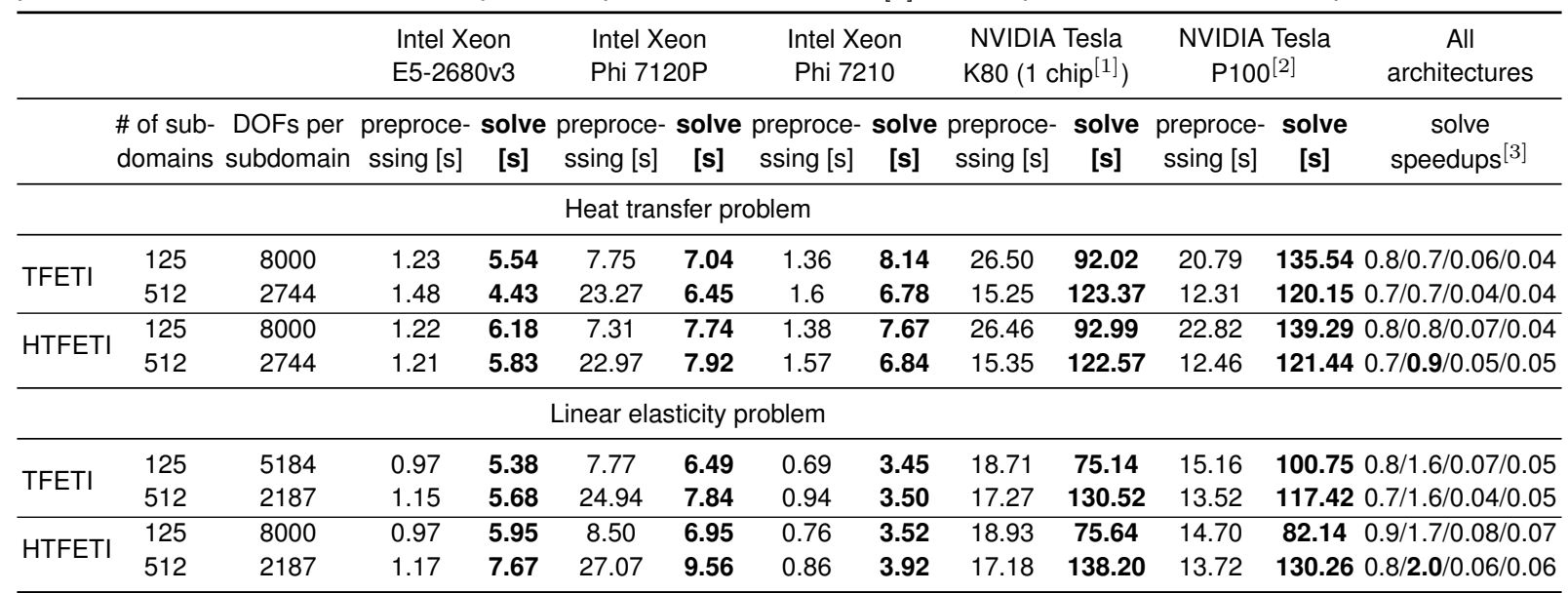

Table 4. Performance of the local Schur complement method on selected architectures. Solve times are measured for 100 iterations of the PCPG solver. Note: [1] with respect to MKL PARDISO sparse solve on CPU.

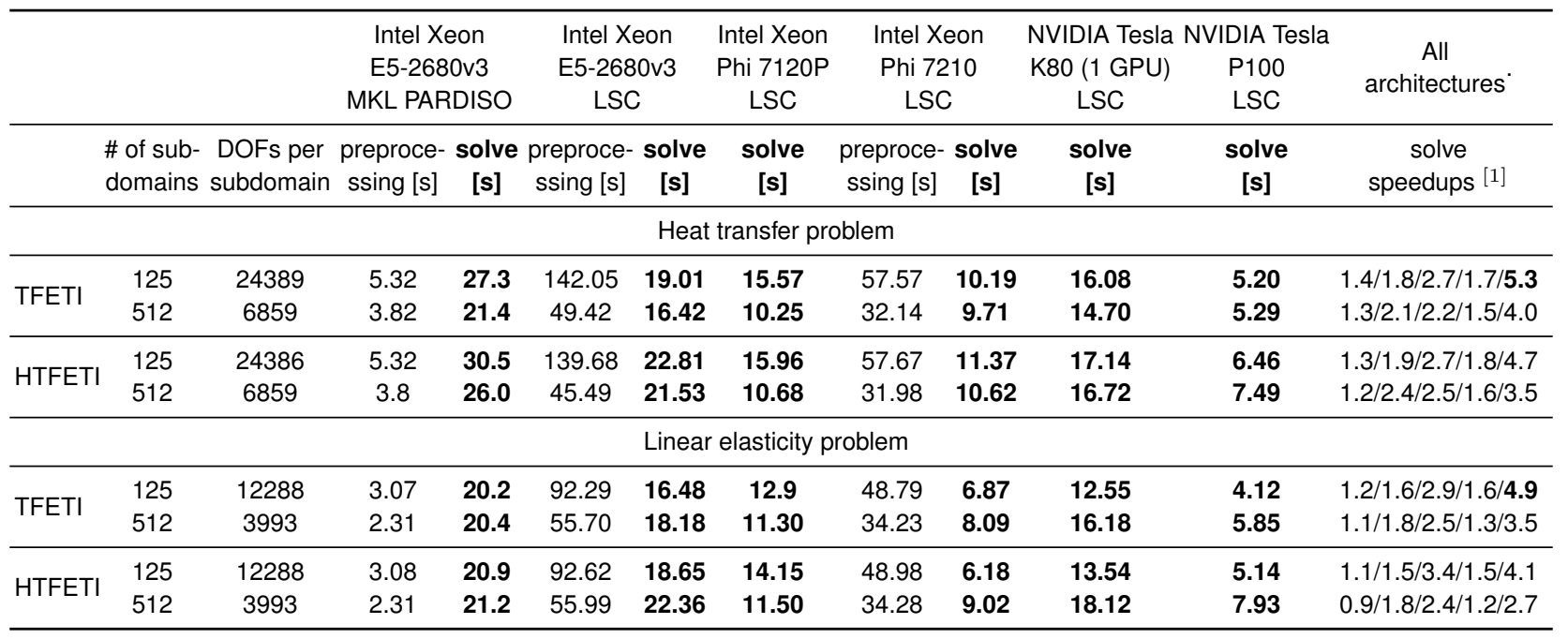

we are using approximately $560 \mathrm{~TB}$ of the CPU memory (we cannot utilize the GPU memory now). The size can potentially be 3 times greater when executed on machines like Sequoia with 1.57 PB of memory (Lawrence Livermore National Laboratory, LLNL, 2017) or the K computer with 1.41 PB of memory (RIKEN Advanced Institute for Computational Science, AICS , 2017).

Large scale memory efficient evaluation. Figure 8 (bottom) shows the weak scalability evaluation of the HTFETI solver for the 3D heat transfer problem. This test uses the iterative solver technique presented in the previous 


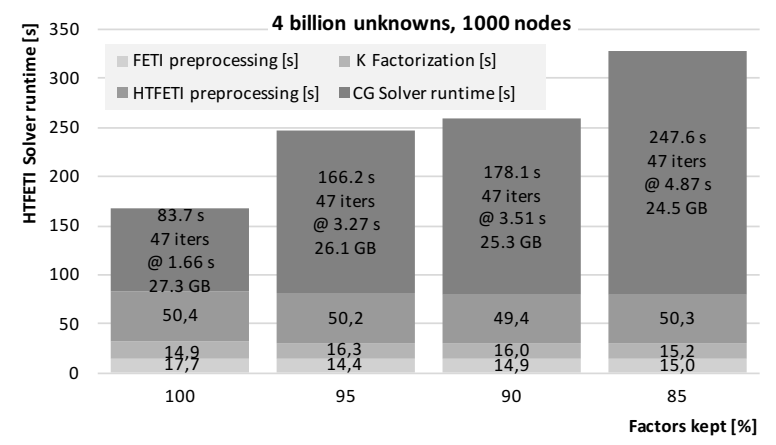

Figure 4. Evaluation of the repeated factorization technique on 1000 nodes of the OLCF Titan machine for 3D linear elasticity.

Table 5. The maximum problem that can be solved on the Titan supercomputer with ESPRESO. Note: [1] Depends on the condition number of subdomain matrices.

\begin{tabular}{lcc}
\hline $\begin{array}{l}\text { Heat Transfer problem } \\
\text { (Laplace equation in 3D) }\end{array}$ & $\begin{array}{c}\text { single } \\
\text { iteration } \\
\text { time [s] }\end{array}$ & $\begin{array}{c}\text { the largest } \\
\text { possible problem } \\
\text { [billion unknowns] }\end{array}$ \\
\hline Factorization in preprocessing & 2.5 & 124 \\
\hline Factorization + LSC on GPU & 3.5 & 136 \\
\hline Iterative solver technique & $18^{[1]}$ & 223 \\
\hline Repeated factorization & 34 & 223 \\
\hline
\end{tabular}

section. One can see that the CG iterative solver takes up the majority of time and we have significantly longer periteration time, approximately $18 \mathrm{~s}$, which is caused by the HTFETI operator. With respect to the scalability, we can see that both parallel scalability (see single iteration time), and numerical scalability (see number of iterations for different problem sizes), perform as expected and are very good.

Finally Table 5 shows a comparison of the presented techniques and (i) a general FETI approach (Cholesky decomposition in preprocessing), which is able to solve 124 billion unknowns, (ii) the combination of the previous approach plus utilization of the GPU memory by LSC, which increases the size of solvable problem by $10 \%$, and (iii) the presented techniques themselves, which can solve 223 billion unknowns.

To sum up, we can state that the proposed memory efficient technique allows us to solve 1.8 times larger problems (124 vs 223 billion unknowns) on the same supercomputer. However, the cost is 7.2 to 13.6 times slower solution time $(2.5 \mathrm{~s}$ vs $18 \mathrm{~s}-34 \mathrm{~s})$, depending on how well conditioned the subdomain matrices are.

\section{Improving energy efficiency using dynamic tunning}

In this section we present how to reduce the energy consumption of a supercomputer while running the ESPRESO library. Our approach is based on dynamicly tuning selected hardware parameters, called tuning parameters, during the application runtime. The tuning parameters are: (i) the CPU core frequency using Dynamic Voltage and Frequency Scaling (DVFS), (ii) the CPU uncore frequency ${ }^{\dagger}$ (Intel Corporation, 2012) and (iii) the number of OpenMP threads. Tuning itself is not performed by ESPRESO, but using a set of external tools. The concept of dynamic tuning is introduced by the
H2020 READEX (2018) project and is described in more by

Schuchart et al. (2017).

In short, the process of dynamic tunning is performed in following three steps:

- Step 1: identify and annotate the significant regions of an application (see Figure 5 for regions that have been used for tuning of the ESPRESO library).

- Step 2: find the optimal settings for the tuning parameters for each of these regions to minimize energy consumption ${ }^{\ddagger}$.

- Step 3: dynamically switch to the optimal settings of tuning parameters during the application runtime.

Step 1 is done manually by a code developer. The goal is to identify the regions with different workloads (compute bound, memory bound, I/O bound, communication bound, etc.) and annotate them in the source code. Annotated regions, also called significant regions, are evaluated in step 2, which is called the analysis phase. During the analysis, the application is executed multiple times with all combinations of the tuning parameters to find the optimal settings for each significant region.

After manual annotation, the analysis is performed automatically using our in-house tools MERIC (performs parameter tuning and energy measurements) and RADAR (analyzes the measurements and creates the tuning model), see Vysocky et al. (2017). The optimal settings are then saved into a file called tunning model, which is used in step 3. Finally, the production runs are done in step 3, in which the dynamic tuning is performed by the MERIC tool. At application startup, MERIC reads the tunning model from the file, and at the beginning of each significant region it changes the tunning parameters to the optimal settings for that particular region. At the end of the region the settings are changed back to the optimal ones for the parent region.

We evaluated the effect of dynamic tunning on the Salomon supercomputer equipped with two Intel Xeon E52680v3, two Intel Xeon Phi 7120p, and 128GB RAM per node (IT4Innovations National Supercomputing Centre, IT4I, 2017). The potential of this approach is presented in Table 6. Results were measured using a heat transfer problem with 3.5 million DOF generated by the mesh generator (see Figure 6 d.). We used this rather small problem to show that this approach can (i) save energy; up to $33 \%$; (ii) reduce processing time; up to $22 \%$; and (iii) improve the strong scalability. Please note, that for 1 to 16 nodes, the energy savings are paid for with extra processing time. However for 32 to 128 nodes both energy and time savings are achieved due to fact that the workload per node is significantly smaller, most of the data fits into L3 caches, and some significant regions use a smaller number of cores.

\footnotetext{
†The uncore region of the Intel Xeon E5-2600v3 CPUs include QPI controllers, L3 cache, a snoop agent pipeline, an on-die memory controller, etc.

${ }^{\ddagger}$ The energy consumption of the CPU and DDR memory is measured using Intel RAPL counters at rate of 1000 samples per second. The energy consumption of the remaining hardware of the compute node is used as a baseline. The baseline of a compute node of the IT4I Salomon supercomputer is between $70-85 \mathrm{~W}$ based on node utilization. We use a simple linear model to estimate the baseline based on the energy consumption of its CPUs.
} 


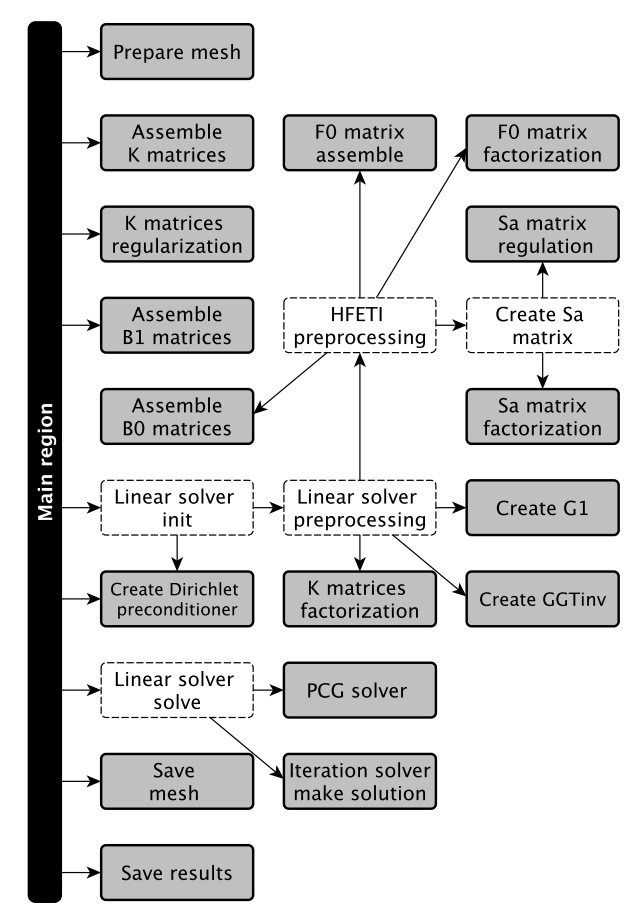

Figure 5. Significant regions of the ESPRESO library used for dynamic tuning in order to reduce the energy consumption.

Table 6. Energy savings analysis for the strong scalability test of the ESPRESO library when running the cube benchmark (see Figure 6 d.).

\begin{tabular}{ccccccc}
\hline & \multicolumn{2}{c}{ no tuning } & \multicolumn{2}{c}{ dynamic tuning } & \multicolumn{2}{c}{ total savings } \\
\hline $\begin{array}{c}\text { number } \\
\text { of nodes }\end{array}$ & $\begin{array}{c}\text { time } \\
{[\mathrm{s}]}\end{array}$ & $\begin{array}{c}\text { energy } \\
{[\mathrm{kJ}]}\end{array}$ & $\begin{array}{c}\text { time } \\
{[\mathrm{s}]}\end{array}$ & $\begin{array}{c}\text { energy } \\
{[\mathrm{kJ}]}\end{array}$ & $\begin{array}{c}\text { time } \\
{[\%]}\end{array}$ & $\begin{array}{c}\text { energy } \\
{[\%]}\end{array}$ \\
\hline 1 & 129.3 & 37.2 & 143.7 & 34.3 & -11.1 & $\mathbf{8 . 0}$ \\
2 & 68.6 & 39.8 & 75.5 & 36.5 & -10.1 & $\mathbf{8 . 2}$ \\
4 & 33.2 & 38.0 & 35.6 & 34.3 & -7.2 & $\mathbf{9 . 8}$ \\
8 & 21.5 & 49.6 & 22.9 & 44.7 & -6.8 & $\mathbf{9 . 9}$ \\
16 & 13.4 & 60.8 & 14.3 & 53.5 & -6.3 & $\mathbf{1 2 . 1}$ \\
32 & 7.7 & 62.2 & 7.2 & 50.6 & 6.1 & $\mathbf{1 8 . 7}$ \\
64 & 4.0 & 69.9 & 3.6 & 52.4 & 9.3 & $\mathbf{2 5 . 0}$ \\
128 & 3.6 & 119.6 & 2.8 & 80.1 & 22.2 & $\mathbf{3 3 . 0}$ \\
\hline
\end{tabular}

\section{Parallel scalability results}

In this section we present large scale tests generated from a built-in mesh generator which is able to generate extremely large meshes with geometric decomposition (cubical problems decomposed into cubical domains, see Figure $6 \mathrm{~d}$ ). This tool is used for all huge scalability tests of the HTFETI solver in this paper because it is able to generate multi-billion unknown problems in tens of seconds. In addition we use three real-world problems generated by Ansys and ELMER.

\subsection{Large Scale Tests with Mesh Generator}

We used two types of problems/physics for scalability evaluation: heat transfer (3D Laplace equation) and 3D linear elasticity, both widely used in structural mechanics. Please note, that in order not to consume too many core-hours, the stopping criteria for the PCPG solver was set to $10^{-3}$ for all large scale tests.

Large Scalability Tests. We evaluated both the strong and weak scalability of the HTFETI solver on up to 17,576 compute nodes of the Titan supercomputer. Figure 7 shows the strong scalability for a 20 billion unknowns heat transfer problem and an 11 billion unknowns linear elasticity problem, respectively. The figure shows that due to the numerical properties of the HTFETI solver, the number of iterations decreases as problem size per compute node/MPI process/HTFETI cluster decreases. Therefore we can state that due to the combination of numerical and parallel scalability in our implementation, we have achieved superlinear scaling.

Figure 8 demostrates the weak scalability of the heat transfer problem on the full OLCF Titan machine. The top chart uses all PARDISO for CPU memory and LSC for GPUs (causing longer preprocessing times) and solves up to 136 billion unknowns. The bottom chart uses the memory efficient iterative solver technique, and it is able to solve up to 223 billion unknowns. In both cases we can observe almost flat scalability. Figure 9 shows a similar comparison for 3D linear elasticity. The top chart is the smallest problem test but has the fastest solution, and uses only PARDISO on the CPU. The middle chart is the test using PARDISO on the CPU and LSC on the GPU, which increases the maximum solvable problem size to 91 billion unknowns. If a repeated factorization technique with $90 \%$ of factors kept in memory is applied, the maximum solvable problem size is 100 billion unknowns (bottom chart). In none of these tests do we use an accelerator to increase the performance, but rather to increase the problem size.

\subsection{Real World Structural Mechanics Problems}

The Ansys Workbench Benchmark. To evaluate the solver on complex geometry we created a 300 million unknowns benchmark shown in Figure 6 a). It is a 3D elasticity benchmark with the following material properties: Young's modulus $E=2 \times 10^{11} \mathrm{~Pa}$, Poisson's ration $\mu=0.3$, density $\rho=4900 \mathrm{~kg} / \mathrm{m}^{3}$, loaded by standard Earth gravity $g=$ $9.8066 \mathrm{~ms}^{-2}$ and additional external forces. The same configuration is also used for other linear elasticity benchmarks.

We decomposed the mesh into 512, 1024, 2048, 4096, and 8192 clusters. Each MPI process owns one cluster and there are 22 MPI processes per node. We used the Salomon supercomputer mentioned in Section 5.

We compared TFETI and HTFETI to show that for a smaller number of MPI processes, TFETI with better convergence is more efficient. Due to the limited parallel scalability of TFETI (caused by the coarse problem) HTFETI is more efficient for larger numbers of clusters.

Intel Xeon Phi Acceleration. The model used for this benchmark is a 120 million unknowns linear elasticity problem shown in Figure 6 b). Here we compare the performance of two Xeon E5-2680v3 and two Xeon Phi 7120 p per node. The CPU is using MKL PARDISO, while the MIC is using LSC. The Dirichlet preconditioner is also accelerated. The table presents two experiments with decompositions into 128 and 512 subdomains per MPI rank. The optimal decomposition is into 128 subdomains, and speedup is 2.04 times. For these tests, load balancing between the CPU and the accelerator was enabled.

The ELMER FEM Benchmarks. For this test we used the Winkel benchmark, which is part of the ELMER 

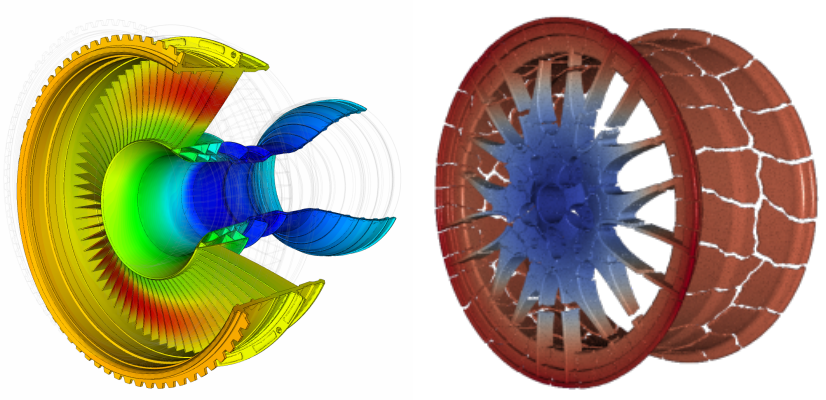

a) Ansys benchmark - scalability tests b) Ansys benchmark - accelerator tests
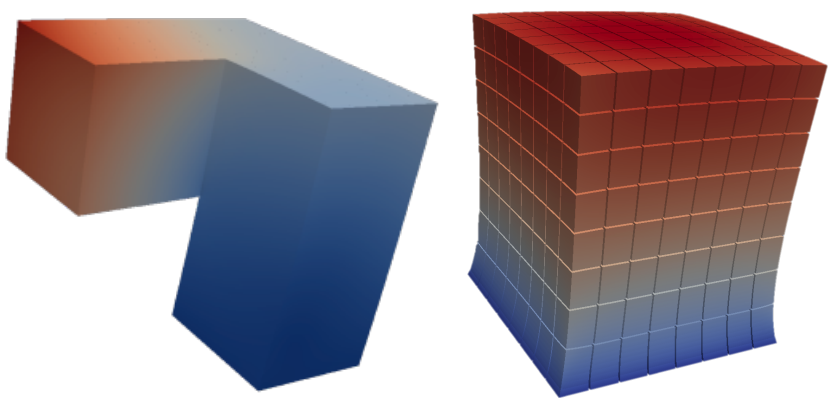

c) ELMER benchmark - scalability tests d) Benchmark generator problem

Figure 6. A set of benchmark problems used in this paper for structural mechanics. Real world problems generated by Ansys Workbench a) a 300 million unknowns problem; b) a 120 million unknowns problem and scalability benchmarks c) the Winkel benchmark in ELMER and d) the Massively Parallel Benchmark from ESPRESO.

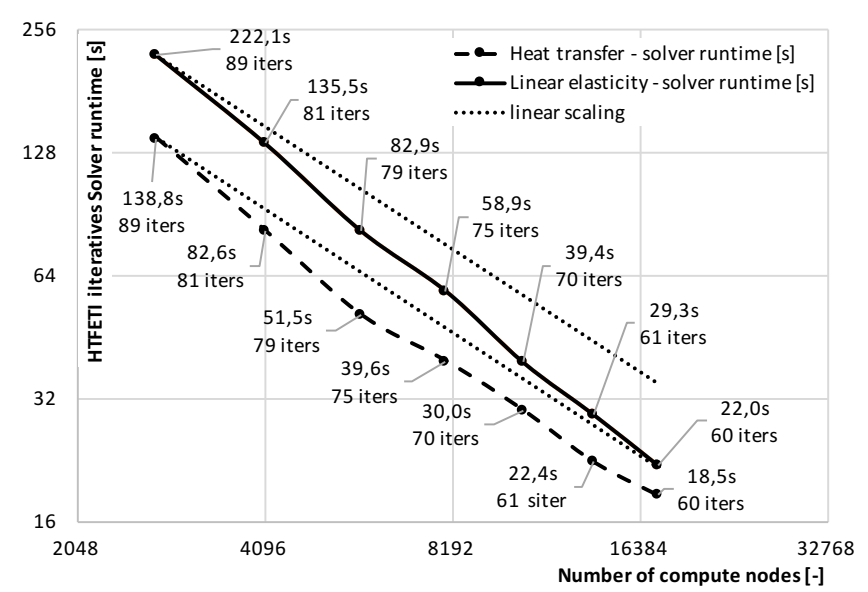

Figure 7. Results of the strong scalability test for heat transfer and linear elasticity problems with 20 and 11 billion unknowns.

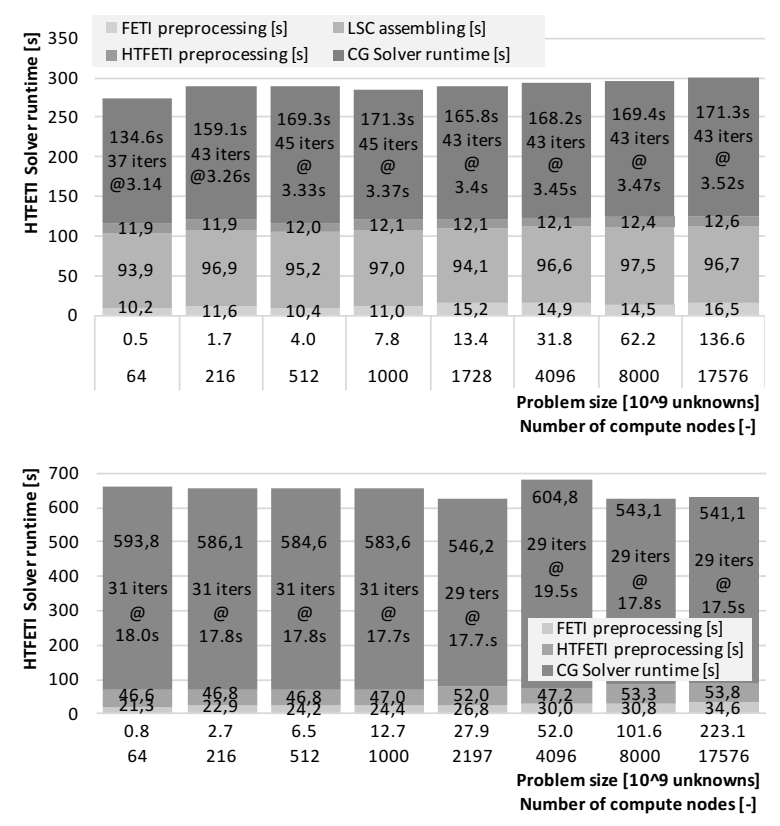

Figure 8. Results of the weak scalability test for the heat transfer problem. Top: a problem wih up to 130 billion unknowns solved by a combination of PARDISO on the CPU and LSC on the GPU. Bottom: a problem with 223 billion unknowns solved using the iterative solver for subdomain matrices.
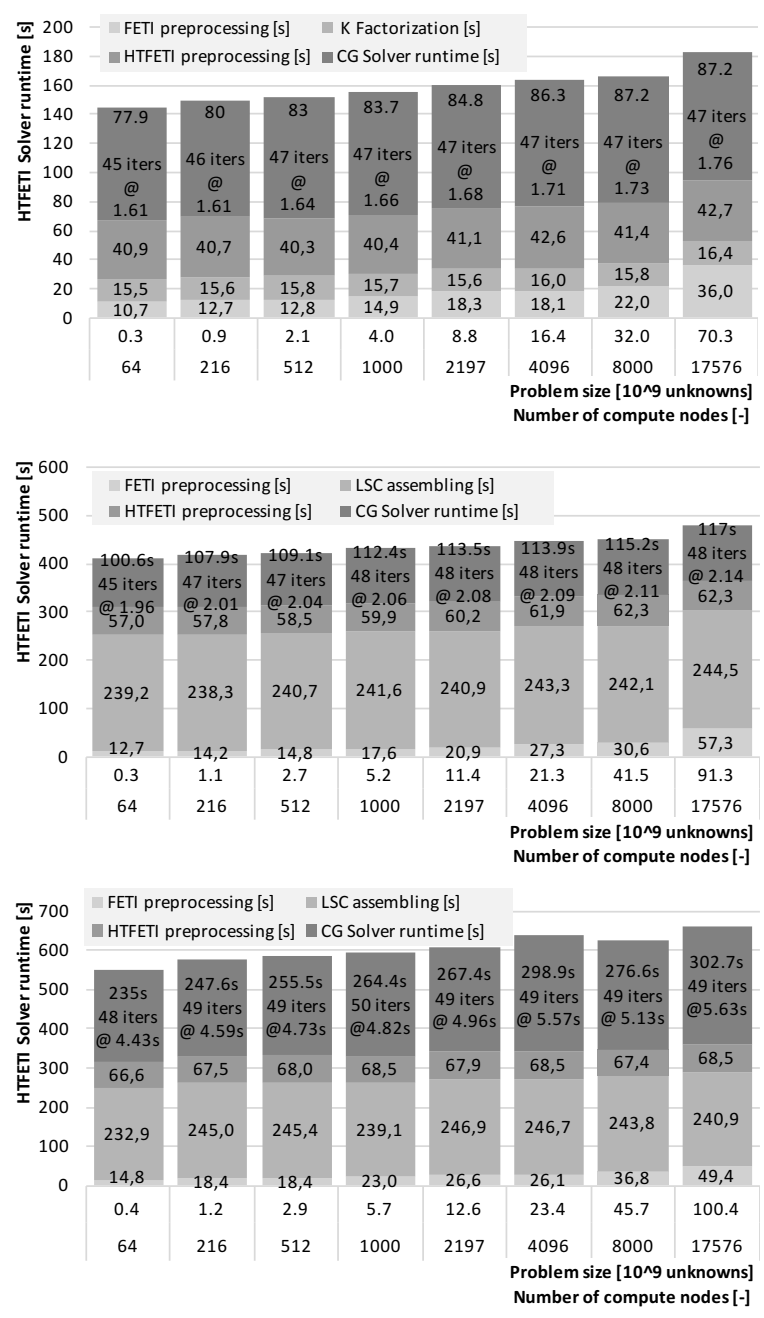

Figure 9. Results of the weak scalability evaluation for the linear elasticity problem with up to 70,92 and 100 billion unknowns. Top: PARDISO only; middle: PARDISO and LSC on the GPU; bottom: PARDISO with repeated factorization (90\%) and LSC on the GPU.

Table 7. Results of the performance evaluation of the Intel Xeon Phi acceleration of the real world problem.

\begin{tabular}{ccccc}
\hline $\begin{array}{c}\text { \# of domains } \\
\text { per MPI rank }\end{array}$ & $\begin{array}{c}\text { \# of } \\
\text { iterations }\end{array}$ & $\begin{array}{c}\text { solver runtime } \\
\text { E5-2680v3 }[\mathrm{s}]\end{array}$ & $\begin{array}{c}\text { solver runtime } \\
\text { Phi 7120p }[\mathrm{s}]\end{array}$ & speedup \\
\hline 128 & 295 & $\mathbf{8 8}$ & $\mathbf{4 2 . 9}$ & $\mathbf{2 . 0 4}$ \\
512 & 363 & 96.5 & 54.9 & 1.78 \\
\hline
\end{tabular}




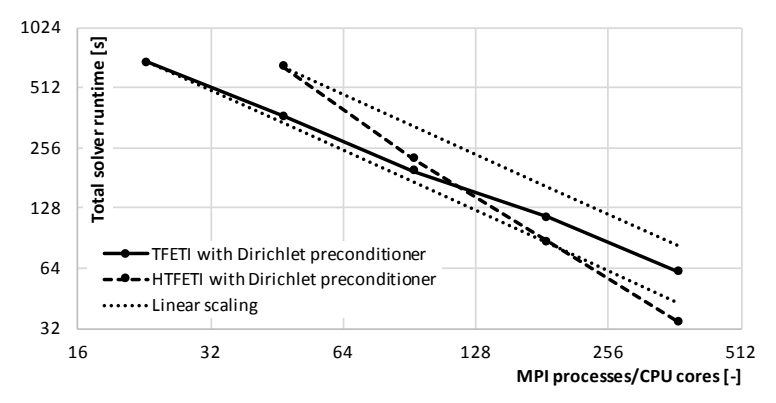

Figure 10. Results of the strong scalability evaluation for a 3D linear elasticity problem with 300 million unknowns, see Figure 6 a).

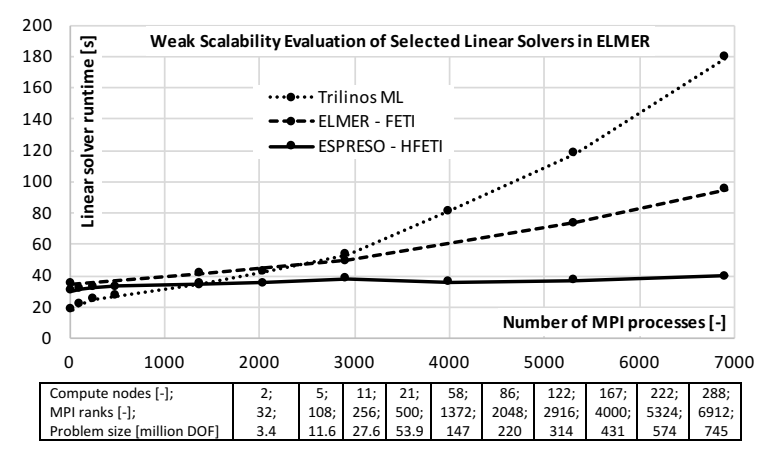

Figure 11. Results of the weak scalability evaluation for 3D linear elasticity problems with up to 745 million unknowns generated by ELMER, including a comparison of the HTFETI solver in ESPRESO, the FETI solver in ELMER, and the ML multigrid solver from Trilinos. All solvers are called directly from ELMER.

package. The large problem size was achieved by the mesh-multiplication technique implemented in ELMER. We compared our HTFETI solver with the FETI solver implemented in ELMER, and the ML multigrid solver from Trilinos (Heroux et al., 2005). For smaller problems the Trilinos ML solver is faster. However, the scalability of the HTFETI solver is better for large problems. The results are shown in Figure 11, with achieved speedup of 4.5 times with respect to Trilinos ML, and 2.45 times with respect to ELMER FETI.

\section{Conclusions}

This paper presents the ESPRESO library and its FEM preprocessing package as well as the modern HTFETI solver. We have demonstrated techniques that can utilize the fast HBM memory of the latest NVIDIA Tesla P100, bringing a speedup of 5.3x for TFETI / 4.7x for HTFETI and 4.9x for TFETI / 4.1x for HTFETI for heat transfer (the 3D Laplace equation) and linear elasticity respectively, when compared to Intel Haswell Xeon E5-2680v3. The difference between the Tesla P100 and Xeon Phi 7210 is mainly caused by different memory bandwidths, since the bandwidth of the $\mathrm{P} 100$ is 1.8 times higher. We also have to take into account that the 7210 must do all the work, while the P100 has a CPU to perform some tasks.

An essential part of our HTFETI solver is its numerical and parallel scalability. The solver achieved superlinear strong scaling from 2744 compute nodes to 17,576 compute nodes for both linear elasticity and heat transfer problems.
We have also presented new techniques that push the boundaries of the HTFETI solver towards larger problems with unstructured meshes. The two techniques can increase the problem size that can be solved by a factor of 1.8 , to 223 billion unknowns on 17,576 nodes with $560 \mathrm{~TB}$ of memory. These approaches have been demonstrated using weak scalability tests.

Using the ESPRESO FEM tools we can solve real-world problems with complex meshes from Ansys Workbench, ELMER, and OpenFOAM. We have demonstrated the superlinear scalability with a combination of TFETI and HTFETI solvers. For these problems, we have also presented the load-balancing technique, and the accelerated Dirichlet preconditioner.

We have also shown that by dynamicly tuning the hardware parameters the ESPRESO library can reduce energy consumption by up to $33 \%$, and save up to $22 \%$ of runtime, while also improving its strong scalability.

\section{Acknowledgements}

This research used resources of the Oak Ridge Leadership Computing Facility at the Oak Ridge National Laboratory, which is supported by the Office of Science of the U.S. Department of Energy under Contract No. DE-AC05-00OR22725, and was supported by a grant from the Swiss National Supercomputing Centre (CSCS) under projects ID d66 and d70, and by the Intel Corporation under the grant 'Intel Parallel Computing Center at IT4Innovations National Supercomputing Center'.

\section{Declaration of conflicting interests}

The Authors declare that there is no conflict of interest.

\section{Funding}

This work was supported by The Ministry of Education, Youth and Sports from the National Programme of Sustainability (NPU II) project IT4Innovations excellence in science - LQ1602 and by the IT4Innovations infrastructure which is supported from the Large Infrastructures for Research, Experimental Development and Innovations project IT4Innovations National Supercomputing Center LM2015070.

This work was supported by the READEX project - the European Unions Horizon 2020 research and innovation programme under grant agreement No. 671657.

The work was also partially supported by the SGC grant No. SP2017/167 Numerical methods for environmental process modelling and No. SP2017/165 Efficient implementation of the boundary element method III, VŠB - Technical University of Ostrava, Czech Republic.

\section{References}

Amestoy P, Davis T and Duff I (1996) An approximate minimum degree ordering algorithm. SIAM JOURNAL ON MATRIX ANALYSIS AND APPLICATIONS 17(4): 886-905. DOI:10. 1137/S0895479894278952.

ANSYS (2017) ANSYS Workbench.

Badia S, Martin A and Principe J (2014) A highly scalable parallel implementation of balancing domain decomposition 
by constraints. SIAM Journal on Scientific Computing 36(2): C190-C218. DOI:10.1137/130931989. URL https://www.scopus.com/inward/record. uri?eid=2-s2.0-84899618766\&doi=10 . $1137 \% 2 \mathrm{f} 130931989 \&$ partner $I D=40 \& \mathrm{md} 5=$ $1 \mathrm{~d} 1 \mathrm{dbbc} 9 \mathrm{fada} 1 \mathrm{dc} 8 \mathrm{eb} 9 \mathrm{f} 5 \mathrm{~b} 00 \mathrm{e} 836 \mathrm{fb} 64$.

Cited By 13 .

Casadei A, Faverge M, Lacoste X and Ramet P (20082017) Parallel Sparse matriX package. http://pastix. gforge.inria.fr.

Dostál Z, Horák D and Kučera R (2006) Total FETI-an easier implementable variant of the FETI method for numerical solution of elliptic PDE. Communications in Numerical Methods in Engineering 22(12): 1155-1162. DOI:10.1002/ cnm.881. URL http://dx.doi.org/10.1002/cnm. 881.

Farhat C, Mandel J and Roux FX (1994a) Optimal convergence properties of the FETI domain decomposition method. Computer Methods in Applied Mechanics and Engineering 115: 365-385. DOI:10.1016/0045-7825(94)90068-X.

Farhat C, Mandel J and Roux FX (1994b) Optimal convergence properties of the FETI domain decomposition method. Comput Method Appl Mech Eng 115: 365-385.

Gosselet P and Rey C (2006) Non-overlapping domain decomposition methods in structural mechanics. Archives of Computational Methods in Engineering 13(4): 515-572. DOI: 10.1007/BF02905857.

Heroux MA, Bartlett RA, Howle VE, Hoekstra RJ, Hu JJ, Kolda TG, Lehoucq RB, Long KR, Pawlowski RP, Phipps ET, Salinger AG, Thornquist HK, Tuminaro RS, Willenbring JM, Williams A and Stanley KS (2005) An overview of the trilinos project. ACM Trans. Math. Softw. 31(3): 397-423. DOI: http://doi.acm.org/10.1145/1089014.1089021.

Intel Corporation (2003-2017) Intel Math Kernel Library. https : //software.intel.com/en-us/intel-mkl.

Intel Corporation (2012) Intel xeon processor e5-2600 product family uncore performance monitoring guide. https://www.intel.com/content/dam/www/ public/us/en/documents/design-guides/ xeon-e5-2600-uncore-guide.pdf.

Intel Corporation (2017a) Intel Xeon E5-2689v3. https://ark.intel.com/products/81908/

Intel-Xeon-Processor-E5-2680-v3-30M-Cache-2 $50-\mathrm{GHz}$.

Intel Corporation (2017b) Intel Xeon Phi 7120p. https://ark.intel.com/products/75799/ Intel-Xeon-Phi-Coprocessor-7120P-16GB-1_ 238-GHz-61-core.

Intel Corporation (2017c) Intel Xeon Phi 7210. https: //ark.intel.com/products/94033/ Intel-Xeon-Phi-Processor-7210-16GB-1_ 30-GHz-64-core.

IT Center for Science, CSC (2017) Elmer. https: / / www . cSc . $\mathrm{fi} / \mathrm{web} / \mathrm{elmer/elmer.}$

IT4Innovations National Supercomputing Centre, IT4I (2017) Salomon supercomputer. https://docs.it4i.cz/ salomon/introduction/.

Jasak H, Jemcov A, Tukovic Z et al. (2007) OpenFOAM: A C++ library for complex physics simulations. In: International workshop on coupled methods in numerical dynamics, volume
1000. IUC Dubrovnik, Croatia, pp. 1-20.

Jeffers J and Reinders J (2013) Intel Xeon Phi Coprocessor High Performance Programming. 1 edition. Morgan Kaufmann. ISBN 9780124104143.

Jeffers J and Reinders J (2014) High Performance Parallelism Pearls Volume One: Multicore and Many-core Programming Approaches. Elsevier Science. ISBN 9780128021996.

Jeffers J and Reinders J (2015) High Performance Parallelism Pearls Volume Two: Multicore and Many-core Programming Approaches. Elsevier Science. ISBN 9780128038901.

Klawonn A, Lanser M and Rheinbach O (2016a) Fe2ti: Computational scale bridging for dual-phase steels. Advances in Parallel Computing 27: 797806. DOI:10.3233/978-1-61499-621-7-797. URL

https://www.scopus. com/inward/record.

uri?eid=2-s2.0-84969913450\&doi=10.3233\% 2 f978-1-61499-621-7-797\&partner $I D=40 \& \mathrm{md} 5=$ ae60853a9101d7bad22126f1481cced4. Cited By 0.

Klawonn A, Lanser M and Rheinbach O (2016b) A highly scalable implementation of inexact nonlinear feti$\mathrm{dp}$ without sparse direct solvers. Lecture Notes in Computational Science and Engineering 112: 255264. DOI:10.1007/978-3-319-39929-4_25. URL https://www. scopus.com/inward/record. uri?eid=2-s2.0-84998887069\&doi=10.1007\% 2f978-3-319-39929-4_25\&partner $I D=40$ \&md5= 0f08ae34d8312345333833ee14f128ac. Cited By 0.

Klawonn A and Rheinbach O (2010) Highly scalable parallel domain decomposition methods with an application to biomechanics. ZAMM 90(1): 5-32. DOI:10.1002/zamm. 200900329. URL http://dx.doi.org/10.1002/ zamm. 200900329.

Kuzmin A, Luisier M and Schenk O (2013) Fast methods for computing selected elements of the Greens function in massively parallel nanoelectronic device simulations. In: Wolf F, Mohr B and Mey D (eds.) Euro-Par 2013 Parallel Processing, Lecture Notes in Computer Science, volume 8097. Springer Berlin Heidelberg. ISBN 978-3-642-40046-9, pp. 533-544. DOI: 10.1007/978-3-642-40047-6_54. URL http://dx.doi. org/10.1007/978-3-642-40047-6_54.

Lawrence Livermore National Laboratory, LLNL (2017) Sequoia supercomputer. http://computation.llnl.gov/ computers/sequoia.

NVIDIA Corporation (2006-2017) NVIDIA CUDA Toolkit. https://developer.nvidia.com/cuda-toolkit.

NVIDIA Corporation (2017a) Nvidia Tesla K20X. http: //www.nvidia.com/content/PDF/kepler/ Tesla-K20X-BD-06397-001-v05.pdf.

NVIDIA Corporation (2017b) Nvidia Tesla K80. http: / / www . nvidia.com/object/tesla-k80.html.

NVIDIA Corporation (2017c) Nvidia Tesla P100. http: / / www . nvidia.com/object/tesla-p100.html.

Oak Ridge Leadership Computing Facility, OLCF (2017) Titan supercomputer. https://www.olcf.ornl.gov/ computing-resources/titan-cray-xk7/.

READEX (2018) Horizon 2020 READEX project. https : // www. readex.eu.

Rennich SC, Stosic D and Davis TA (2016) Accelerating sparse cholesky factorization on gpus. Parallel Computing 59: 140 - 150. DOI:http://dx.doi.org/10.1016/j.parco.2016. 
06.004. URL http://www.sciencedirect.com/ science/article/pii/s016781911630059x.

Riha L, Brzobohaty T, Markopoulos A, Meca O and Kozubek T (2016a) Massively Parallel Hybrid Total FETI (HTFETI) Solver. In: Platform for Advanced Scientific Computing Conference, PASC. ACM. ISBN 978-1-4503-4126-4/16/06. DOI:DOI:http://dx.doi.org/10.1145/2929908.2929909.

URL http://espreso.it4i.cz/wp-content/ uploads/2016/05/RIHA_PASC2016.pdf.

Riha L, Brzobohaty T, Markopoulos A, Meca O, Kozubek T, Schenk O and Vanroose W (2016b) Efficient Implementation of Total FETI Solver for Graphic Processing Units using Schur Complement. In: HPCSE 2015, LNCS 9611. DOI:DOI:10.1007/978-3-319-40361-86. URL http://espreso.it4i.cz/wp-content/ uploads/2016/05/HPCSE2015-GPU.pdf.

RIKEN Advanced Institute for Computational Science, AICS (2017) K computer. http://www. aics.riken.jp/en/ k-computer/system.

Schenk O, Bollhöfer M and Römer RA (2008) On largescale diagonalization techniques for the Anderson model of localization. SIAM Rev. 50(1): 91-112. DOI:10. 1137/070707002. URL http: / / dx.doi.org/10.1137/ 070707002 .

Schenk O, Wächter A and Hagemann M (2007) Matchingbased preprocessing algorithms to the solution of saddle-point problems in large-scale nonconvex interior-point optimization. Computational Optimization and Applications 36(2-3): 321341. DOI:10.1007/s10589-006-9003-y. URL http: // dx . doi.org/10.1007/s10589-006-9003-y.

Schuchart J, Gerndt M, Kjeldsberg PG, Lysaght M, Horák D, Říha L, Gocht A, Sourouri M, Kumaraswamy M, Chowdhury A, Jahre M, Diethelm K, Bouizi O, Mian US, Kružík J, Sojka R, Beseda M, Kannan V, Bendifallah Z, Hackenberg D and Nagel WE (2017) The readex formalism for automatic tuning for energy efficiency. Computing 99(8): 727-745. DOI: 10.1007/s00607-016-0532-7. URL https://doi.org/ 10.1007/s00607-016-0532-7.

Shroeder W, Martin K and Lorensen B (1998) The visualization toolkit. Prentice Hall 24: 25.

Sistek J and Cirak F (2015) Parallel iterative solution of the incompressible navier-stokes equations with application to rotating wings. Computers and Fluids 122: 165183. DOI:10.1016/j.compfluid.2015.08.026. URL https://www. scopus.com/inward/record. uri?eid=2-s2.0-84942244914\&doi=10.1016\% $2 \mathrm{fj}$. compfluid.2015.08.026\&partner $I D=40$ \& md5 $=c 785182 a 8 c 6 c 4432506 e 31481 b 95 d 1 f f . \quad$ Cited By 2.

Vysocky O, Beseda M, Riha L, Zapletal J, Nikl V, Lysaght M and Kannan V (2017) Evaluation of the HPC Applications Dynamic Behavior in Terms of Energy Consumption. In: Ivanyi P, Topping BHV and Varady G (eds.) Proceedings of the Fifth International Conference on Parallel, Distributed, Grid and Cloud Computing for Engineering. Civil-Comp Press, Stirlingshire, UK. Paper 3, 2017. doi:10.4203/ccp.111.3.

\section{Author Biographies}

Lubomir Riha is head of the Infrastructure Research Lab at IT4Innovations National Supercomputing Centre (IT4I). Previously he was a senior researcher in the Parallel Algorithms Research Lab at IT4I and a research scientist in High Performance Computing Lab at The George Washington University, ECE Department. He has received his Ph.D. and M.Sc. degrees in Electrical Engineering from Czech Technical University in Prague, Czech Republic in 2011 and D.Sc. degree in Computer Science from Bowie State University, USA. He was investigator of FP7 EXA2CT project and Intel Parallel Computing Center and principal investigator of the H2020 READEX project at IT4I. He is also a co-principal developer of the ESPRESO library. His research interests are high performance computing, energy efficient computing, acceleration of scientific and engineering applications using multi- and manycore accelerators, development of scalable linear solvers, signal and image processing.

Michal Merta is a researcher at IT4Innovations National Supercomputing Center. He obtained his $\mathrm{PhD}$. from Computational and Applied Mathematics at VSB - Technical University of Ostrava for a research on a parallelization of the time-dependent boundary element method for the wave equation. He is a co-founder of the library of boundary element method based solvers BEM4I and has participated in several national and international projects, including the Intel Parallel Computing Center at IT4Innovations focused on optimization for multi- and many- core architectures, or a bi-lateral Czech-Austrian project Efficient Parallel Implementations of Boundary Element Methods. In 2015, he received the Joseph Fourier Prize for research in the field of computational sciences awarded by Bull and the Embassy of France in the Czech Republic. $\mathrm{He}$ is a co-author of more than 20 articles and conference papers.

Radim Vavrik is a Ph.D. student in Computational Sciences and research assistant in the Infrastructure Research Lab at IT4Innovations National Supercomputing Center. He is mainly interested in parallel computing, scalable algorithms design, GPU acceleration and code optimization, and heterogeneous architectures. He worked on hydrological and flood modeling software, and high-performance heterogeneous platform for energy efficient computing. Now he is working on GPU acceleration of the ESPRESO library.

Tomas Brzobohaty is a senior researcher at IT4Innovations and has Ph.D. degree in Applied mechanics. His research interest includes development of scalable algorithms for large-scale engineering problems. He is one of the principal developers of highly parallel solvers implemented in the ESPRESO library for current and future supercomputers. He initiated and sustained collaborations with most IT4I industrial partners (Continental, Skoda Doosan Power, MAVEL). He participated at IPCC (Intel Parallel Computing Center) and several EC funded projects such as READEX, EXPERTISE, CLOUDIFACTURING, and TETRAMAX. He is an author or co-author of more than 30 publications including papers in journals with impact factor.

Alexandros Markopoulos is a postdoctoral fellow at UPMC, Paris 6 currently working on coupled problems arising within the elastohydrodynamic lubrication contact. Previously he was a researcher at IT4Innovations National Supercomputing Center, Ostrava. His research interests focus on the finite element method, the algorithms for large scale problems based on domain decomposition methods and the Krylov subspace methods. He was involved in the implementation of FETI based solvers in ESPRESO 
library. He is an author or co-author of more than 40 scientific articles with 15 of them published in prestigious journals.

Ondrej Meca is a researcher at IT4Innovations National Supercomputing Center. $\mathrm{He}$ is also a Ph.D. student working towards a degree in Computer Science at VSB - Technical University of Ostrava. His research interests include verification of parallel algorithms and the implementation of scalable algorithms for large-scale engineering problems. He is responsible for the implementation of the pre- and post-processing parts of the ESPRESO library.

Ondrej Vysocky received his M.Sc. degree in Computer Science from the Brno University of Technology, Czech Republic in 2016. His master thesis focused on parallel I/O optimization. Currently he is a Ph.D. student at VSB - Technical University of Ostrava, Czech Republic. His research is focused on energy-efficiency in high performance computing. $\mathrm{He}$ is also an investigator of the Horizon 2020 READEX project which deals with energy efficiency of HPC applications using dynamic tunning.

Tomas Kozubek is the Scientific Director at IT4Innovations National Supercomputing Center. He specializes in Scalable Algorithms for Solving Extensive Problems of Mechanics, Exascale Algorithms and Advanced Techniques of Parallelization, FETI- based Domain Decomposition Methods, Solvers for
Quadratic Programming Problems, Reliability and Robustness of Solutions. He has earned his Ph.D. degree in Informatics and Applied Mathematics at VSB - TU Ostrava for his doctoral thesis called Numerical Solution to Partial Differential Equations using Efficient Fast Solvers. Tomas Kozubek is a coordinator of work teams within the Partnership for Advanced Computing in Europe (PRACE) international project. He organizes a series of workshops and conferences focused on HPC. Besides that he is an author or co-author of more than 60 scientific articles, at least 30 of which have been published in prestigious impact factor journals.

Vit Vondrak is the Managing Director at IT4Innovations National supercomputing center and associated professor at VSB - Technical University of Ostrava. His expertise is in numerical linear algebra, optimization methods, high performance computing. He is the member of the PRACE Council (Partnership for Research and Advanced Computing in Europe) representing the Czech Republic. $\mathrm{He}$ is/was co-investigator of 4 FP7 projects PRACE-1IP, PRACE2IP, PRACE-3IP, HARPA and H2020 projects PRACE-4IP and PRACE-5IP. Leader of mathematical modelling group in the project FLOREON+ (http://floreon.eu) - system for disaster management support. Principal investigator of the project Intel Parallel Computing Center and project of the Large infrastructures of the Czech republic. Vit Vondrak is author of more than 60 scientific papers, 7 papers in journals with impact factor, h-index 4 on WoS. 